DRAFT VERSION MARCH 2, 2022

Preprint typeset using LTEX style emulateapj v. 11/10/09

\title{
EXTERNAL MASS ACCUMULATION ONTO CORE POTENTIALS: IMPLICATIONS FOR STAR CLUSTERS, GALAXIES AND GALAXY CLUSTERS
}

\author{
J. P. NAIMAN ${ }^{1}$, E. RAMIREZ-RUIZ ${ }^{1}$ AND D. N. C. LIN ${ }^{1}$ \\ Draft version March 2, 2022
}

\begin{abstract}
Accretion studies have been focused on the flow around bodies with point mass gravitational potentials, but few general results are available for non-point mass distributions. Here, we study the accretion flow onto nondivergent, core potentials moving through a background medium. We use Plummer and Hernquist potentials as examples to study gas accretion onto star clusters, dwarf and large galaxy halos and galaxy clusters in a variety of astrophysical environments. The general conditions required for a core potential to collectively accrete large quantities of gas from the external medium are derived using both simulations and analytic results. The consequences of large mass accumulation in galaxy nuclei, dwarf galaxies and star clusters are twofold. First, if the gas cools effectively star formation can be triggered, generating new stellar members in the system. Second, if the collective potential of the system is able to alter the ambient gas properties before the gas is accreted onto the individual core members, the augmented mass supply rates could significantly alter the state of the various accreting stellar populations and result in an enhanced central black hole accretion luminosity.

Subject headings: accretion, accretion disks, globular clusters:general, hydrodynamics, galaxies:general
\end{abstract}

\section{INTRODUCTION}

The process by which stars gravitationally capture ambient matter is called accretion. Calculations of the accretion flow onto stars are, in general, very difficult. First, one must determine the flow geometry; in general, if the gas possesses intrinsic angular momentum, it will be multidimensional depending upon the flow symmetry. Second, one must enumerate the dominant heating and cooling mechanisms that characterize the accreting plasma. If the gas is optically thick to the emitted radiation, the net cooling and heating rates will depend on the radiation field. Third, the effect of radiation pressure in holding back the flow must be properly accounted for. Fourth, one must understand the flow boundary conditions, both at large distances and at the surface of the accretor. Fifth, the possible role of magnetic fields in the plasma must be assessed. So, it is not surprising that the problem of gas accretion onto stars has been solved in only a few idealized cases. In spite of the difficulties, significant progress has been achieved in understanding accretion flows by means of such idealized solutions.

The problem of accretion of gas by a star in relative motion with respect to the gas was first considered by Hoyle and Lyttleton (1939) and later by Bondi and Hoyle (1944) (BHL accretion). The spherically symmetric case, when the accreting mass is at rest with respect to the ambient gas, was first studied by Bondi (1952) and is commonly referred to as Bondi accretion. This gives a reasonable approximation to the real situation of an isolated star accreting from the interstellar medium, provided that the angular momentum, magnetic field strength and bulk motion of the interstellar gas with respect to the star can be neglected. For other types of accretion flows, such as those in close binary systems, spherical symmetry is rarely a good approximation. Nonetheless, the spherical accretion problem is of great relevance for the theory, as it introduces some key concepts which have much broader validity. What is more, it is possible to give a fairly exact treatment, allowing

\footnotetext{
${ }^{1}$ Department of Astronomy and Astrophysics, Theoretical Astrophysics Santa Cruz, University of California, Santa Cruz, CA 95064; jnaiman@ucolick.org
}

us to gain insight into more complicated situations.

Accretion studies have been focused on the flow around bodies with point mass gravitational potentials, but few general results are available for non-point mass distributions. Mass models with tractable potentials may accurately approximate the potentials of galaxies, galaxy clusters and dense stellar systems. In this paper we consider calculations of the gas flow onto these smooth core potentials. We are interested in determining the amount of mass accumulation for core potentials with a range of velocities relative to the ambient gas. In $\S 2$, we briefly recapitulate the relevant equations which describe the gas structure and deduce analytically the conditions for generalized core potentials to accumulate gas collectively. Because the gas structure is multi-dimensional in nature, we adopt a numerical approach to verify our analytic solutions. We describe the numerical scheme and the range of model parameters in $\S 3$, and the results of these calculations in $\S 4$. These results are applied, in $\$ 5$, to study the accumulation of gas into dense stellar systems, galaxies and galaxy clusters moving in a variety of environments. In $\$ 6$, we summarize our results and discuss their implications.

\section{MASS ACCUMULATION IN A CLUSTER CORE POTENTIAL}

\subsection{Generalized Solutions}

Much of the effort herein will be dedicated to understanding the conditions by which smooth core potentials are able to accrete surrounding gas with highly enhanced rates. In particular, we would like to understand under which conditions a stable density enhancement will persist within a given potential well and derive a relation between the gas relative velocity, the local sound speed and the the central velocity dispersion of the cluster potential, which can be carried over quite generally to more complicated accretion flows.

To treat the problem mathematically we proceed as in Lin \& Murray (2007). We take spherical polar coordinates with origin at the center of the potential. In spherical symmetry, the fluid variables are independent of angle and the gas velocity $u$ has only a radial component. For a steady flow, the continuity 
equation reduces to

$$
r^{2} \rho u=\text { constant. }
$$

This constant is commonly denoted to as $\dot{M}$, and refers to a mass inflow (outflow) rate. In the Euler equation the only contribution to the external force comes from gravity and this has only a radial component

$$
u \frac{d u}{d r}+\frac{1}{\rho} \frac{d P}{d r}+\frac{d \phi}{d r}=0 .
$$

Hence, the term $(1 / \rho)\left(\frac{d P}{d r}\right)$ is $\left(c_{\mathrm{s}}^{2} / \rho\right)(d \rho / d r)$, where $c_{\mathrm{s}}$ is the sound speed. We replace the energy equation by the polytropic relation

$$
P=K \rho^{\gamma}, \quad K=\text { constant }
$$

In this case: $c_{\mathrm{s}}^{2}=d P / d \rho=K \gamma \rho^{\gamma-1}$. This allows us to approximately treat both adiabatic $(\gamma=5 / 3)$ and isothermal $(\gamma=1)$ gas flows. After the solution has been found, the adiabatic or isothermal assumption should be justified by consideration of the particular radiative cooling and heating of the gas.

The gravitational potential of dense stellar systems, galaxies and galaxy clusters can be accurately described by smooth core potentials with

$$
\phi(r)=-\frac{\phi_{0}}{\left(r^{\beta}+\bar{\varpi}_{\mathrm{c}}^{\beta}\right)^{\alpha}}=-\frac{\phi_{0}}{\zeta(r)},
$$

where $\bar{\sigma}_{c}$ is the radius of the core. The potential's core scale is given by

$$
\zeta(r)=\left(r^{\beta}+\bar{\sigma}_{\mathrm{c}}^{\beta}\right)^{\alpha}
$$

Cores potentials described by (4) are non-divergent, so that $\phi(r \mapsto 0)=$ constant. This property, as we will show, allows core potentials to sustain subsonic inflow and collect ambient gas into a quasistatic envelope.

An estimate of the radial density distribution of collected ambient gas, $\rho(r)$, follows from the application of the conservation laws for energy and momentum. From equation (2) we have

$$
\frac{u^{2}}{2}+\int \frac{d p}{\rho}+\phi=\frac{u^{2}}{2}+\frac{c_{\mathrm{s}}^{2}}{\gamma-1}+\phi=\text { const. }
$$

Equation (6) makes use of the polytropic equation of state, where $d p=K \gamma \rho^{\gamma-1} d \rho$ and $c_{\mathrm{s}}^{2}=\gamma p / \rho=K \gamma \rho^{\gamma-1}$. Here, the limits $\phi(r \mapsto \infty)=0$ and $u(r \mapsto \infty)=0$ help determine the constant

$$
\frac{u^{2}}{2}+\frac{c_{\mathrm{s}}^{2}}{\gamma-1}+\phi=\frac{c_{\infty}^{2}}{\gamma-1}
$$

Dividing equation (7) by $c_{\mathrm{s}}^{2}$ and making use of $c_{\mathrm{s}}^{2} / c_{\infty}^{2}=$ $\rho^{\gamma-1} / \rho_{\infty}^{\gamma-1}$, one recovers a modified form of the Bernoulli equation in Bondi (1952)

$$
\frac{1}{2} \frac{u^{2}}{c_{\mathrm{s}}^{2}}+\frac{1}{\gamma-1}\left[1-\left(\frac{\rho_{\infty}}{\rho}\right)^{\gamma-1}\right]=-\frac{\phi}{c_{\infty}^{2}}\left(\frac{\rho}{\rho_{\infty}}\right)^{\gamma-1}
$$

The isothermal version of the Bernoulli equation can be derived from equation (6) using $d p=K d \rho$ and $c_{\mathrm{s}}=c_{\infty}$,

$$
\frac{1}{2} \frac{u^{2}}{c_{\mathrm{s}}^{2}}+\log \rho+\frac{\phi}{c_{\infty}^{2}}=\log \rho_{\infty}
$$

Together with knowledge of the radial velocity profile, equation (8) allows one to determine $\rho(r)$. For subsonic inflow $u^{2}(r) / c_{\mathrm{s}}^{2} \ll 1$, the ambient gas collects into a a quasistatic envelope with a density distribution given by

$$
\rho(r) \approx \rho_{\infty}\left[1+\frac{(\gamma-1) \phi_{0}}{c_{\infty}^{2} \zeta(r)}\right]^{\frac{1}{\gamma-1}},
$$

where $\rho_{\infty}$ is the density of the ambient medium. For an isothermal equation of state, this result is simply

$$
\rho(r)=\rho_{\infty} \exp \left[\frac{\phi_{0}}{c_{\infty}^{2} \zeta(r)}\right]
$$

In $\$ 2.1 .1$, we derive under what conditions we can safely ignore the $u(r)^{2} / c_{\mathrm{s}}^{2}$ term in equation 10 .

\subsubsection{Subsonic Flows in Core Potentials}

We now consider the validity of the subsonic inflow assumption $|u(r)|<c_{\mathrm{S}}$ used to derive equation (10) assuming subsonic flow at infinity, $\left|u_{\infty}\right|<c_{\infty}$. In spherically symmetry, the radial structure of the flow's velocity can be calculated by integrating (2) with the help of (3) and (1), so that

$$
\frac{1}{2}\left(1-\frac{c_{\mathrm{s}}^{2}}{u^{2}}\right) \frac{d\left(u^{2}\right)}{d r}=\frac{1}{r}\left(2 c_{\mathrm{s}}^{2}-r \frac{d \phi}{d r}\right)
$$

At large distances from the potential the term $\left(2 c_{\mathrm{s}}^{2}-r \frac{d \phi}{d r}\right)$ on the right hand side of equation 12 must be positive since $c_{\mathrm{s}}^{2}$ approaches $c_{\infty}^{2}$ and $\phi \propto 1 / r$, while $r$ increases without limit. Given the that the inflow is initially subsonic, it will remain subsonic provided

$$
c_{\mathrm{s}}^{2}>\frac{r}{2} \frac{d \phi}{d r}
$$

for all $r$ in the flow such that there is no transonic point.

Because $c_{\mathrm{s}}$ is a monotonically decreasing function of $r$, equation 13 is satisfied provided

$$
c_{\infty}^{2}>\frac{r}{2} \frac{d \phi}{d r} .
$$

Consequently, condition (14) guarantees $|u(r)|<c_{\mathrm{s}}$ throughout the gas flow.

Describing $\phi(r)$ as in equation (4), condition (14) can be rewritten as

$$
c_{\infty}^{2}>\frac{1}{2} \frac{\beta \alpha r^{\beta} \phi_{0}}{\left(r^{\beta}+\bar{\omega}_{\mathrm{c}}^{\beta}\right)^{\alpha+1}} .
$$

The right hand side of equation $(15)$ has a maximum at

$$
r=\left(\frac{\bar{\omega}_{\mathrm{c}}}{\alpha}\right)^{1 / \beta}
$$

so that condition (14) is satisfied for core potentials if

$$
c_{\infty}^{2}>\frac{\phi_{0}}{\bar{\omega}_{\mathrm{c}}^{\beta \alpha}} \frac{\beta}{2}\left(\frac{\alpha}{\alpha+1}\right)^{\alpha+1} .
$$

Notice that as $\bar{\omega}_{\mathrm{c}} \mapsto 0$, the ambient gas is unable to collect into a quasistatic envelope and, as a result, the density structure of the flow is no longer accurately described by equation (10). In this limit, the potential essentially reduces to that of a point mass and the inflow rate can be derived following Bondi (1952). 


\subsubsection{Mass Accumulation in Non-Stationary Core Potentials}

The density structure of ambient gas collecting into a core potential in the case that the gravitational structure is collecting gas isotropically is described by equation (10). If the potential is moving with some velocity with respect to the ambient medium, one expects the build up of a stable density enhancement to be inhibited. A characterization of this impedance on the growth of the central density enhancement is derived here by comparing the pressure support due to the collected ambient gas to that provided by the ram pressure. The radius at which these two pressures are equal defines a characteristic radius, which we denote here as $r_{\mathrm{s}}$. A schematic diagram illustrating the geometry of the flow around non-stationary core potentials, together with a sketch of the expected sound speed and density profiles of the collected ambient material, is given in Figure 1.

The characteristic radius, $r_{\mathrm{s}}$, is calculated here under the assumption that a stable density enhancement has been established, whose radial profile is well described by equation (10). The potential, together with its collected ambient gas, is then assumed to move with a constant velocity, $u_{\text {const }} \approx u_{\infty}=\mu_{\infty} c_{\infty}$. This provides a reasonable approximation provided that the flow variables across the shock region are accurately described in terms of their limiting values (i.e. the incoming flow is not significantly altered by the gravitational potential and therefore the interior flow preserves its static solution). When this high velocity gas hits the stationary ambient medium, a bow shock forms. In the frame moving with the shock, the density jump condition can be written as

$$
\frac{\rho_{\mu}}{\rho_{\infty}}=\frac{(\gamma+1) \mu_{\infty}^{2}}{(\gamma+1)+(\gamma-1)\left(\mu_{\infty}^{2}-1\right)}
$$

where $\rho_{\mu}=\rho(r)$, with $\rho(r)$ given by equation $(10)$. Equation (18) assumes the adiabatic index $\gamma$ is the same for the post and pre-shock gas. The jump conditions across the shocked region yield

$$
\zeta\left(r_{\mathrm{s}}\right)=\frac{\phi_{0}(\gamma-1)}{c_{\infty}^{2}}\left[\left\{\frac{(\gamma+1) \mu_{\infty}^{2}}{2+(\gamma-1) \mu_{\infty}^{2}}\right\}^{\gamma-1}-1\right]^{-1}
$$

where we have used $\zeta\left(r_{\mathrm{s}}\right)=\left(r_{\mathrm{s}}^{\beta}+\bar{\varpi}_{\mathrm{c}}^{\beta}\right)^{\alpha}$. These standoff distance define the characteristic radius of the bow shock. For $\phi_{0} \propto M$ we have $\alpha \cdot \beta=1$, so that

$$
\zeta\left(r_{\mathrm{s}}=\bar{\varpi}_{\mathrm{c}}\right) \equiv 2^{1 / \alpha} \bar{\varpi}_{\mathrm{c}} .
$$

Equation (19) can be rewritten for an isothermal equation of state as

$$
\zeta=\frac{\phi_{0}}{c_{\infty}^{2}} \frac{1}{\log \mu_{\infty}^{2}} \quad \text { for } \quad \gamma \rightarrow 1
$$

In general, core potentials moving at high velocities have density enhancements and characteristic radii that decrease with increasing velocity. At sufficiently high Mach numbers, one expects the incoming flow's ram pressure to overcome the central pressure due to the collected ambient gas and to penetrate into the potential's core. The transition between a stable density enhancement and an unsupported, supersonic inflow occurs roughly when $r_{\mathrm{s}} \approx \Phi_{\mathrm{c}}$. When $r_{\mathrm{s}} \leq \bar{\varpi}_{\mathrm{c}}$, the ability of the core to sustain a stable density enhancement is lost. For this reason, we expect rapidly moving potentials to have smaller density enhancements than those predicted by equation 10 . In these cases our formalism inherently overesti- mates the internal pressure support against the inflowing ambient material, which in turn overestimates the characteristic radius. The detailed behavior of the characteristic radius with adiabatic index, Mach number and gravitational potential type will be explored in $\$ 3$ using numerical simulations. In the following numerical models, we will approximate isothermal equations of state by setting $\gamma=1.01$. For consistent comparison, we will use equations $(10)$ and 20 with $\gamma=1.01$ in all plots unless otherwise noted.

\subsection{Specific Core Potentials}

In this paper we focus our attention on the ability of stellar clusters, galaxies, and galaxy clusters to collect ambient material. Stellar clusters can be accurately described by Plummer potentials (Brüns et al. 2009, Pflamm-Altenburg \& Kroupa 2009)

$$
\phi(r)=\frac{G M_{\mathrm{p}}}{\left(r^{2}+r_{c}^{2}\right)^{1 / 2}},
$$

where $M_{\mathrm{p}}$ is the mass of the potential and $r_{\mathrm{c}}$ is the commonly referred to as core radius. In this case, $\phi_{0}=G M_{\mathrm{p}}, \varpi_{\mathrm{c}}=r_{\mathrm{c}}$, $\beta=2$, and $\alpha=1 / 2$, so that equation $(10)$ can be rewritten as

$$
\rho(r)=\rho_{\infty}\left[1+\frac{G M_{\mathrm{p}}(\gamma-1)}{c_{\infty}^{2}\left(r^{2}+r_{\mathrm{c}}^{2}\right)^{1 / 2}}\right]^{\frac{1}{\gamma-1}}
$$

Dark matter profiles of galaxies and galactic clusters are, on the other hand, commonly modeled by Hernquist (1990) potentials

$$
\phi(r)=\frac{G M_{\mathrm{h}}}{r+a},
$$

where $M_{\mathrm{h}}$ is the mass of the potential and $a$ is the scale length. In these cases, $\phi_{0}=G M_{\mathrm{h}}, \bar{\varpi}_{c}=a, \beta=1$, and $\alpha=1$, so that equation 10 becomes

$$
\rho(r)=\rho_{\infty}\left[1+\frac{G M_{\mathrm{h}}(\gamma-1)}{c_{\infty}^{2}(r+a)}\right]^{\frac{1}{\gamma-1}}
$$

In what follows, these two types of potentials are numerically modeled moving through ambient gas at rest to and compare to our analytic treatment.

\section{SIMULATIONS OF MASS ACCUMULATION IN CORE POTENTIALS}

\subsection{Numerical Method and Initial Model}

To examine the ability of core potentials to collect ambient gas into a quasistatic envelope, we simulate core potentials moving through ambient gas with FLASH, a parallel, adaptive mesh refinement hydrodynamics code. This scheme, and tests of the code are described in Fryxell et al. (2000).

Common to all calculations is the placement of inflow boundaries to one side of our computational domain in order to simulate the motion of the core potential through the external medium. As a result, ambient gas moves to the right of our grid at a supersonic velocity. When this high velocity gas hits the stationary potential, a bow shock forms. All gravitational potentials are modeled here using a Plummer 22) or a Hernquist (24) functional form.

Simulations start with a uniform background density and run until a steady density enhancement is established inside the core's potential. This usually takes about 10-100 sound crossing times (Naiman et al. 2009). Several models were run longer to test convergence and density enhancements were 
found to change only slightly with longer run times. We further tested convergence of our models for several resolutions and domain sizes. All tests produced similar density enhancements to those shown here. After hundreds of sound crossing times, the flow is relatively stable, and does not exhibit the flip-flop instability seen in two dimensional simulations (Blondin \& Pope 2009).

The effects of self gravity of the gas are ignored. This is adequate for most of our models, for which the accumulated mass is significantly less than the mass responsible for the potential. To improve the controlled nature of the models, and to facilitate comparison with our analytic estimates, we do not explicitly include radiative heating or cooling. As with our analytic estimates, we assume a polytropic equation of state $P \propto \rho^{\gamma}$. The effects of radiative equilibrium are approximated by having the gas evolve with an adiabatic constant $\gamma=1.01$ (giving nearly isothermal behavior), while inefficient cooling is model here by having the gas evolve with $\gamma=5 / 3$.

\subsection{General Properties of the Gas Flow}

The classical Bondi treatment for non-divergent potentials, representative of star cluster and galactic gravitational potentials, is only a fair approximation far from the core when $G M_{\mathrm{c}} / \bar{\omega}_{\mathrm{c}} \gg c_{\infty}^{2}+u_{\infty}^{2}$. When $G M_{\mathrm{c}} / \bar{\varpi}_{\mathrm{c}} \lesssim c_{\infty}^{2}+u_{\infty}^{2}$, the collective potential alters the local gas properties, allowing the core to sustain subsonic mass inflow and, as a result, collect ambient gas into a quasistatic envelope.

Figure 2 shows the resulting density structures of the collected ambient gas for a point mass and two different core potentials. To facilitate comparison, the Plummer and Hernquist potentials depicted in Figure 2 have been constructed to yield the same circular velocity peak radius: $r_{\mathrm{v}}$. This requirement can be rewritten as

$$
a=\sqrt{2} r_{\mathrm{c}}
$$

which, by demanding the mass within this characteristic radial scale to be the same, gives

$$
M_{\mathrm{h}}=\frac{8}{3} \sqrt{\frac{2}{3}} M_{\mathrm{p}} .
$$

Hence, equations (23) and (25) give

$$
\frac{\rho_{\mathrm{h}}(r=0)}{\rho_{\mathrm{p}}(r=0)} \approx\left(\frac{8}{3 \sqrt{3}}\right)^{\frac{1}{\gamma-1}},
$$

where $\rho_{\max } \equiv \rho(r=0) \gg \rho_{\infty}$.

The flow pattern around a core potential is multidimensional and complex. In the frame of the potential, the gas streamlines are bent towards the cluster center. Some shall intersect the center, while others converge along a line behind it. The convergence speed of the gas determines the reduction in its velocity relative to the potential due to shocks, and therefore whether or not the gas is accumulated in the core. As seen in Figure 2, the flow structure within the cluster markedly differ from the classical Bondi flow.

The properties of the core potential have a decisive effect on the radial profiles of the collected ambient gas. For $r \ll \Phi_{c}$ and $\rho_{\max } \gg \rho_{\infty}$, equation 10 gives

$$
\frac{\rho(r)}{\rho_{\max }} \approx\left[1-\alpha\left(r / \bar{\omega}_{\mathrm{c}}\right)^{\beta}\right]^{\frac{1}{\gamma-1}}
$$

This shows that gas collected in potentials with steep core profiles have, as expected, sharper central density profiles.
From equations 29 and $\sqrt{26}$, it follows that $\rho / \rho_{\max } \propto[1-$ $\left.\left(r / \sqrt{2} r_{\mathrm{c}}\right)\right]^{\frac{1}{\gamma-1}}$ and $\rho / \rho_{\max } \propto\left[1-2\left(r / r_{\mathrm{c}}\right)^{2}\right]^{\frac{1}{\gamma-1}}$ for Hernquist and Plummer potentials, respectively. Figure 2 shows that the the steeper Hernquist interior potential produces gas accumulation which is more centrally confined within its core than that in the Plummer potential.

At $r \gg \Phi_{c}$, the divergence of the gravitational potential naturally leads to a loss of pressure support against the incoming material. As a result, the characteristic radius, $r_{\mathrm{s}}$, will be smaller for less extended (or less massive) core potentials. Inserting equation (27) into equation (19) and making use of equation (20), one finds

$$
\frac{\zeta\left(r_{\mathrm{s}}=\varpi_{\mathrm{c}}\right)_{\mathrm{h}}}{\zeta\left(r_{\mathrm{s}}=\varpi_{\mathrm{c}}\right)_{\mathrm{p}}}=\frac{8}{3 \sqrt{3}}
$$

for Hernquist and Plummer potentials yielding the same circular velocity peak radius, $\gamma, \mu_{\infty}$ and $c_{\infty}$. Under these conditions, the ambient gas collected into a Hernquist potential at $r \gg \bar{\omega}_{\mathrm{c}}$ will be comparatively denser and thus lead to an increase in pressure support against the incoming flow. This is mainly due to the comparatively larger mass (see equations (27) and (19)) together with the less divergent potential at large radii. This enhanced support forces the flow to move around the central density enhancement, thus leading to a widening of the standing bow shock. This is evident in Figure 3. which plots the bow shock patterns produced around a point mass (Ruffert 1994), Plummer and Hernquist potential set in motion through an initially uniform medium.

\subsection{Mach Number Dependence}

In line with the conventional treatment, potential cores moving with respect to the ambient medium at increasing supersonic velocities will have density enhancements that are progressively smaller and significantly more offset from the cluster's center. Figure 4 shows the resulting density and velocity flow structures generated around a point mass, Plummer and Hernquist potential moving through an initially uniform medium with $\mu_{\infty} \geq 1$. The differences in the flow structure are most notable when comparing the flow velocity structures depicted in Figure 4, which show that the central density enhancements in core potentials are quasi-static, while those for the point mass are not. While there are regions of inflowing gas around core potentials, primarily behind the bow shock and downstream from the core's center, there are large central regions that experience no net inward gas flow.

A stable density enhancement is observed to persist as long as $\mu_{\infty} \lesssim 3$ (Figure 4). For larger relative velocities, the central gas pressure support is overwhelmed by the ram pressure of the incoming gas and, as a result, the bow shock begins to detach from the potential's core. The transition from a bow to a tail shock takes place at around $\mu_{\infty} \approx 3$. Note that in the case of a point mass potential with an arbitrarily small sink radius, the gravitational forces will always overwhelm the ram pressure at a finite radius. In this case, there is no transition to a tail shock (Wolfson 1977). However, for core potentials (and point mass potentials with a finite sink size), the gravitational force approaches a constant value near the potential's center, thus allowing the formation of a tail shock.

As shown in Figure 5, the radial density profiles of the accumulated ambient gas, within a core potential differ from the classical Bondi profiles, and, for low Mach numbers, are accurately described by the quasistatic solutions derived in $\$ 2$ These solutions fail to provide a good description of the den- 
sity of the inflowing gas for $\mu_{\infty} \gg 1$. This is because these solutions were derived assuming a static ambient gas. The decrease of the characteristic radius and of the collected ambient gas density with increasing $\mu_{\infty}$ are clearly visible in the simulations. This decrease is connected with an increase of the ram pressure of the incoming material which, in turn, helps overcome the central pressure of the collected ambient gas.

\subsection{Dependence on the Polytropic Index $\gamma$}

The determination of the internal structure of gas flowing around gravitational potentials depends upon detailed knowledge of the equation of state. In $\$ 2$, we have derived analytical solutions for the internal pressure and density structure of the flow in the cases in which the pressure depends solely upon the density through an equation of state of the form $P=K \rho^{\gamma}$.

The dependence of the overall gas density structure on the polytropic index $\gamma$ is illustrated in Figures 6 and 7 . By comparing the flow structures in Figures 6 and 7. one sees that ambient gas is in fact collected much more gradually when its pressure support increases adiabatically $(\gamma=5 / 3)$. From equation 10$\rangle$, it follows that $P \propto \zeta^{\frac{-\gamma}{\gamma-1}}$. Thus, gas accumulated in potentials with higher adiabatic indexes will be less centrally confined and, as a result, will be less effective at protecting the collected gas from being displaced by the inflowing material. By contrast, gas collected isothermally $(\gamma \approx 1)$ into a core potential will be comparatively denser and thus lead to a significant increase in pressure support at small radii. This is consistent with the behavior observed in Figure 6, where decreasing $\gamma$ moves the shock closer to the potential's core. Despite the initially less extended bow shock, the enhanced support provided by more compressible flows at small radii delays the transition to a tail shock as the relative velocity increases and, as a result, the bow shock radius decreases.

\subsection{General Conditions Required for Large Density Enhancements}

A core potential can sustain a large central density enhancement provided that $r_{\mathrm{s}} \gtrsim \bar{\omega}_{\mathrm{c}}$. This condition can be rewritten as $G M / \Phi_{\mathrm{c}} \gtrsim\left(c_{\infty}^{2}+u_{\infty}^{2}\right) \equiv V^{2}$, or alternatively, as $\sigma_{\mathrm{v}} \gtrsim V$ where $\sigma_{\mathrm{v}}$ is the velocity dispersion of the system.

In Figure 8 we examine the ability of a core potential to collect ambient gas as a function of the characteristic radius $r_{\mathrm{s}}$. A density enhancement is observed to persist as long as $r_{\mathrm{s}} \gtrsim \varpi_{\mathrm{c}}$. Once $r_{\mathrm{s}}<\varpi_{\mathrm{c}}$, the collective potential is unable to alter the properties of the flow within $r \leq \Phi_{\mathrm{c}}$.

In addition, the equation of state alters the compressional properties of the incoming flow. Isothermal flows, corresponding to $\gamma \approx 1$, allow for the effective build up of mass. Adiabatic gas, $\gamma=5 / 3$, will instead rapidly heat the gas as it accumulates in the core, and halt the gas inflow at much lower central densities than for the $\gamma \approx 1$ case. Figure $9 \mathrm{com}$ pares the simulated density enhancements results with those predicted by equation 10 .

\subsection{Bow Shock Instabilities}

Most shock instabilities identified and studied in astrophysics are related to the acceleration or deceleration of the shock itself (e.g. Bernstein \& Book 1978, Vishniac 1983). By definition, stationary shocks are stable with respect to these mechanisms, but can still be unstable. Nakayama (1993) pointed out the radial instability of the shock if the flow is immediately accelerated after the shock transition, in isothermal flows. In this respect the shocked Bondi flow should be unstable. The validity of the postshock acceleration criterion for adiabatic flows is still uncertain, even for radial perturbations (Nakayama 1994). Independently of the sign of post-shock acceleration, Foglizzo \& Tagger (2000) and Foglizzo (2001) revealed another generic instability mechanism based on the cycle of acoustic and vortical perturbations in the shock region.

The vortical-acoustic instability is fundamentally nonradial. In this case, both vorticity and entropy perturbations are advected from the shock to the accretor, and both are coupled to the acoustic perturbations. The most unstable cycle involves high frequency acoustic waves, those able to explore the hottest parts of the flow but still be refracted out, with a wavelength slightly larger than the smallest size of the sonic surface. In the isothermal limit $(\gamma \rightarrow 1)$, where entropy perturbations are absent from the problem, vorticity is more appropriate than entropy to describe the advective-acoustic cycle.

The entropic-acoustic cycle is expected to be an efficient instability mechanism for $\gamma \approx 5 / 3$, provided $\mu_{\infty} \gtrsim 2$ and that the distance to the sonic surface (with depends on $r_{\mathrm{s}}$ ) is small enough (Foglizzo et al. 2005). This coincides with our numerical simulations of core accretion which show strong instability when $r_{\mathrm{s}} \approx \Phi_{\mathrm{c}}$ (Figure 8). The lack of strong instability of adiabatic flows around core potentials with $r_{\mathrm{s}} \gg \Phi_{\mathrm{c}}$ could be due to the lack of effective acoustic feedback from the advected vorticity perturbation. Our numerical experiments also show the presence of instabilities within fast moving, isothermal flows. In particular isothermal flows appears much more unstable when $r_{\mathrm{s}} \approx \Phi_{\mathrm{c}}$ (Figure 6). In this conditions, a small pressure perturbation of the shock is able to generate vorticity perturbations very efficiently for $\gamma=1$ strong shocks. The differences seen in the shock geometry between these two limiting cases precludes an accurate comparison of the stability of the flow, especially since the instability threshold is very sensitive to the size and shape of the sonic surface (Foglizzo et al. 2005).

\section{ASTROPHYSICAL RELEVANCE}

The ability of core potentials to collect ambient mass depends sensitively on their velocity dispersion, relative speed with respect to the ambient gas and gas cooling properties. Estimates of velocity dispersion and environmental gas properties are known for a wide range of systems, including star clusters, dwarf galaxies, large galactic halos, and clusters of galaxies. In what follows, we refine our calculations to include a wide variety of embedding media and discuss how environmental properties conspire to enable or disable the effective collection of surrounding mass in these systems. We assume the individual stellar members are accreting at low enough rates such that mass removal (and feedback) can be neglected. These results are summarized in Table 1 .

\subsection{Star Clusters}

Star clusters are generally well described by Plummer potentials (Plummer 1911, Brüns et al. 2009: Pflamm-Altenburg \& Kroupa 2009). In this section, we will discuss two limiting cases spanning the range of environments star clusters might inhabit. First, we discuss a compact star cluster moving slowly with respect to cold gas, representative of young clusters in a galaxy merger environment. This situation is simulated in Figure 10 (model A). Second, we model a less compact cluster moving quickly with respect to the background 
gas. These less favorable conditions for gas accumulation are simulated in Figure 10 (model B).

\subsubsection{Young Star Clusters}

Young massive star clusters are frequently found in merging systems like the Antennae and Cartwheel galaxies (Whitmore et al. 1999, Crivellari et al. 2009). In the Antennae complex, for example, young (compact) star clusters are observed to move slowly with respect to the cold gas: $c_{\infty} \approx 10 \mathrm{~km} \mathrm{~s}^{-1}$ and $\mu_{\infty} \lesssim 2$ (? Gilbert \& Graham 2007). Observations in the infrared suggest large amounts of dust present in the gas (Brandl et al. 2005), which justifies an assumption of isothermal gas. A typical cluster, $M_{\mathrm{p}}=3.5 \times 10^{5} M_{\odot}, \varpi_{\mathrm{c}}=r_{\mathrm{c}}=1 \mathrm{pc}$, in this cold, efficiently cooling gas $(\gamma=1.01)$, moving at $\mu_{\infty}=2.0$ is shown in Figure 10 (model A). In these merging environments, conditions are ideal for accumulating large amounts of ambient gas in these systems as $r_{\mathrm{s}} \gg r_{\mathrm{c}}$. Taking $\rho_{\infty} \approx 10^{-22} \mathrm{~g} \mathrm{~cm}^{-1}$, similar to that inferred by Zhu et al. (2003), we find the collected mass to be a sizable fraction of the total cluster mass: $M_{\mathrm{acc}} / M_{\mathrm{p}} \approx 0.05$.

For these young clusters (< Gyr), one might worry about the applicability of our results, as feedback due to massive stellar winds is neglected in our treatment. We can estimate the magnitude of this effect by comparing the ram pressure from stellar winds of the cluster members to the pressure support generated by the collected ambient gas. The ram pressure due to expanding stellar winds is approximately given by $P_{*}=\rho_{*} v_{*}^{2}=N \dot{M}_{*} v_{*} /\left(4 \pi r^{2}\right)$ for $N$ massive stars. Each star here is assumed to have a mass loss rate and wind velocity $\dot{M}_{*}$ and $v_{*}$, respectively, expanding into a spherical volume of radius $r$. We can then calculate the size of the wind bubble, $r_{\mathrm{w}}$, by equating $P_{*}\left(r_{\mathrm{w}}\right)=P_{\rho}\left(r_{\mathrm{w}}\right)$, where $P_{\rho}=c_{\infty}^{2}[\rho(r)]^{\gamma} / \gamma \approx c_{\infty}^{2}\langle\rho\rangle$, where $\langle\rho\rangle$ is the average density in the cluster's core. Solving for $r_{w}$ gives $r_{\mathrm{w}} \approx 0.02 N_{2}^{1 / 2} \dot{M}_{*,-7}^{1 / 2} v_{*, 3}^{1 / 2}\langle\rho\rangle_{-18}^{-1 / 2} c_{\infty, 1}^{-1} \mathrm{pc}$, where $N=10^{2} N_{2}, \dot{M}_{*}=10^{-7} \dot{M}_{*,-7} M_{\odot} \mathrm{yr}^{-1}, v_{*}=10^{3} v_{*, 3} \mathrm{~km} \mathrm{~s}^{-1}$, $\langle\rho\rangle=10^{-18}\langle\rho\rangle_{-18} \mathrm{~g} \mathrm{~cm}^{-3}$ and $c_{\infty}=10 c_{\infty, 1} \mathrm{~km} \mathrm{~s}^{-1}$. For large density enhancements, we have $r_{\mathrm{w}} \ll r_{\mathrm{c}} \approx 1 \mathrm{pc}$ and, therefore the stellar winds would be unable to significantly impede the inflowing of gas.

\subsubsection{Globular Clusters}

Globular cluster's orbits are primarily dictated by the host galactic potential (Harris 1996, 1991). In the Milky Way, globular clusters (GCs) are observed to move at highly supersonic velocities $\mu_{\infty} \sim 5-10$ (Dinescu et al. 1997). In addition, the gas within the galactic halo is expected to be virialized so that $c_{\infty} \approx u_{\infty}$, implying $V^{2} \gg G M_{\mathrm{p}} / \varpi_{\mathrm{c}}$. As a result, for a typical GC with $M_{\mathrm{p}}=3.5 \times 10^{5} M_{\odot}$ and $\varpi_{\mathrm{c}}=r_{\mathrm{c}}=2 \mathrm{pc}$ moving at $\mu_{\infty}=4.0$, one expects negligible mass accumulation as $r_{\mathrm{s}} \lesssim r_{\mathrm{c}}$. This is true even if the cluster is surrounded by cold, efficiently cooling ambient gas $\left(c_{\infty}=10 \mathrm{~km} \mathrm{~s}^{-1}\right.$ and $\left.\gamma=1.01\right)$ as shown in Figure 10 (model B). In this case, for a background density similar to that of the ISM, $\rho_{\infty} \approx 10^{-24} \mathrm{~g} \mathrm{~cm}^{-3}$, the accumulated mass is only $M_{\text {acc }} / M_{\mathrm{p}} \approx 2 \times 10^{-6}$.

A GC might collect gas more effectively if its orbit happened to lie in the disc plane of its host galaxy. If the GC moving with $\mu_{\infty} \leq 2$ through the galactic disc, we might expect the mass accumulation to more closely resemble that seen for model A in Figure 10 However, these conditions are rare for GCs (Dinescu et al. 1997).

\subsection{Galactic Systems}

Hernquist potentials (Hernquist 1990) are used to describe a wide variety of galactic systems - from the dark matter halos of dwarf galaxies and Milky Way sized systems, to the halos of galaxy clusters (Adén et al. 2009, Scott et al. 2009, Hwang \& Lee 2008). In what follows we assume, for simplicity, that these gravitational potentials are not significantly modified from their dark matter profiles by the presence of gas or stars.

\subsubsection{Dwarf Galaxy Halos}

Dwarf galaxies (DGs) are seen either moving through the IGM or embedded within larger halos (Diemand et al. 2008 Mateo 1998). For those moving under the influence of a larger gravitational potential we expect that, on average, DGs to orbit around the ambient gas with a velocity comparable to the circular velocity of their host: $V^{2} \gtrsim G M_{\mathrm{h}} / a$. However, many DGs are found to reside in nearly radial orbits, and thus will spend only a small fraction of their orbital time moving at lower velocities (Diemand et al. 2008, 2007).

For example, a DG on a highly elliptical orbit, $e=0.9$, will spend approximately $1 \%$ of its orbital period moving at less than $20 \%$ of the host's circular velocity. During this period a large central density enhancement is expected to be effectively sustained within the DG' core if $G M_{\mathrm{h}} / a \gtrsim c_{\infty}^{2}+u_{\infty}^{2}$. That is, if the DG is surrounded by cold gas with $c_{\infty}^{2} \lesssim G M_{\mathrm{h}} / a$. Such an ideal situation for mass accumulation is illustrated by model $\mathrm{C}$ in Figure 11. In these conditions, if the density of cold gas is $\rho_{\infty}=10^{-25} \mathrm{~g} \mathrm{~cm}^{-3}$, the mass collected by the DG could be as large as $M_{\text {acc }} / M_{\mathrm{h}} \approx 0.14$. In contrast, if the surrounding gas is virialized, $c_{\infty} \approx \sigma_{\mathrm{v}, \text { host }} \gg G M_{\mathrm{h}} / a$, the DG halo will spend most of its orbit with mass inflows similar to those depicted for model D in Figure 11. In this case, for $\rho_{\infty}=10^{-25} \mathrm{~g} \mathrm{~cm}^{-3}$, the accumulated mass is only $M_{\mathrm{acc}} / M_{\mathrm{h}} \approx 5 \times 10^{-4}$.

\subsubsection{Halos in Merging Systems}

A massive galactic halo is likely to experience a relatively equal mass merger during its lifetime. Galaxies of $M \approx$ $10^{11} M_{\odot}$ will have undergone, on average, about one merger with a galaxy of $M \gtrsim 5 \times 10^{10} M_{\odot}$ since $z=1.2$ (Robaina et al. 2010). Because the smaller component is moving under the gravitational influence of a halo of similar mass, we expect $u_{\infty} \approx \sigma_{\mathrm{v}}$. Moreover, if gas is effectively virialized within more massive halo, then $c_{\infty} \approx \sigma_{\mathrm{v}}$ and, as a result, a large density build up is expected to be effectively retained in the core of the smaller halo. This situation is depicted in Figure 12 (model E), in which a a Milky Way-like sized halo is simulated moving through hot gas with $c_{\infty}=100 \mathrm{~km} / \mathrm{s}$ at $\mu_{\infty}=2.0$. Comparing the bremsstrahlung cooling and the core sound crossing time, we find $t_{\text {cool }} \gtrsim t_{\text {dyn }}$. Since cooling is expected to be inefficient, we assume $\gamma=5 / 3$. While $r_{s} \gtrsim \Phi_{c}=a$, ineffective cooling results in an accumulated mass of only $M_{\mathrm{acc}} / M_{\mathrm{h}}=10^{-2}$ for ambient densities of $\rho_{\infty}=10^{-27} \mathrm{~g} \mathrm{~cm}^{-3}$.

\subsubsection{Galaxy Clusters}

Similar to the case discussed above, a large central gas density enhancement is expected in equal mass merging galaxy

\footnotetext{
${ }^{2}$ We note here that model $\mathrm{C}$ has been run until a quasi-static density profile has been established, which takes about a few core sound crossing times. If the DG is at apogee for a shorter time, the accumulated mass is expected to be lower.
} 
clusters, such as the Bullet cluster complex. The Bullet cluster is a galaxy cluster merging with another slightly more massive cluster (Markevitch et al. 2002). In this case, $M_{\mathrm{h}}=10^{15} M_{\odot}$, $\bar{\omega}_{\mathrm{c}}=a=1 \mathrm{Mpc}, \mu_{\infty}=2.5$, and $c_{\infty}=1580 \mathrm{~km} \mathrm{~s}^{-1}$. For an inefficiently cooled gas $(\gamma=5 / 3)$, this results in $r_{\mathrm{s}} \approx \bar{\varpi}_{\mathrm{c}}=a$. Such situation is depicted in Figure 13 (model F). Assuming IGM densities of $\rho_{\infty}=10^{-27} \mathrm{~g} \mathrm{~cm}^{-3}$ results in an accumulated mass of $M_{\mathrm{acc}} / M_{\mathrm{h}}=3.5 \times 10^{-4}$. This simple model of the Bullet cluster gives similar flows structures to those found in more sophisticated calculations which include the stellar and gas mass distributions.

\section{DISCUSSION}

In $\$ 4$ we have shown the conditions required to collectively accumulate large quantities of gas from the ambient medium in the centers of a variety of astrophysical systems thought to be well described by a core gravitational potential. The consequences of large mass accumulation in such systems fall into two basic categories. First, if the gas cools effectively star formation can be triggered, generating new stellar members in the system. Second, if the gas accretes efficiently onto the core's current stellar population, the state of these stellar systems (and possibly the central black hole) can be significantly perturbed. In what follows we discuss the implications of gas accumulation and their accompanying observational signatures.

\subsection{The State of the Accumulated Gas \\ 5.1.1. Emission Properties}

The emission properties of the accumulated gas in clusters of galaxies and young stellar clusters are briefly reviewed in this section. At temperatures $\gtrsim 3 \times 10^{7} \mathrm{~K}$, the primary cooling process for intracluster gas is thermal bremsstrahlung, while for temperatures $\lesssim 3 \times 10^{7} \mathrm{~K}$, line cooling becomes very important. For the the intracluster gas in most star clusters, the cooling time is longer than the Hubble time (age of the universe). Thus cooling is not dynamically very important in these cases. Figure 14 (bottom panel) shows the surface brightness profile, calculated assuming optically thin cooling resulting from bremsstrahlung and collisionally excited metal transitions, for a cluster merging environment with parameters selected to mimic the Bullet Cluster complex (model F). We find an integrated bolometric luminosity of $L_{\text {bol }} \approx 10^{44} \mathrm{erg} \mathrm{s}^{-1}$ and a bowshock structure that are comparable to those observed by Chandra (Markevitch 2006 Markevitch et al. 2002). In the center of the cluster, where cooling is important, our calculated emission is considerably harder than found in simulations including the cooling term in the energy equation (Milosavljević et al.2007).

Also shown in Figure 14 is the surface brightness profile of the accumulated gas in a young star cluster with parameters similar to those seen for the stellar cluster members in the Antennae galaxy merging system (model A). To calculate the emission we assume optically thin cooling, where we employ an effective cooling curve. In the cluster's core, the near infrared (NIR) surface brightness is approximately $\approx 10^{41} \mathrm{erg} \mathrm{s}^{-1} \mathrm{pc}^{-2}$, which is probably optimistic given that we do not explicitly include the cooling term in the energy equation but instead use an adiabatic simulation. This simple model gives, nonetheless, results that are consistent with the observed range of luminosities in the Antennae star clusters: $L_{\mathrm{NIR}}=10^{39}-10^{42} \mathrm{erg} \mathrm{s}^{-1}$ (Brandl et al. 2005). For clusters embedded in these types of merging environments, the emission from the bowshock and cluster core should distinguishable ${ }^{3}$ given enough spatial resolution and provided the reprocessed stellar radiation is not the dominant contribution to the NIR.

\subsubsection{Enhanced Central Densities Leading to Star Formation}

If the gas collected by these potentials cools efficiently, we would expect it to fragment and form stars if the density is high enough. In general, one can relate the gas density to the star formation rate (Kennicutt 1998): $\Sigma_{\text {sfr }} \approx 10^{-4}$ $\left(\Sigma_{\text {gas }} / 10^{4} M_{\odot} \mathrm{pc}^{-2}\right)^{1.4} M_{\odot} \mathrm{yr}^{-1} \mathrm{pc}^{-2}$, where $\Sigma_{\text {sfr }}$ and $\Sigma_{\text {gas }}$ are the star formation and gas column densities, respectively. A dwarf galaxy, for example, embedded within a larger halo with properties similar to those assumed for model $\mathrm{C}$ (i.e., $\rho_{\infty}=10^{-24} \mathrm{~g} \mathrm{~cm}^{-3}$ and a $200 \mathrm{pc}$ star forming region) is expected to form stars at a rate of $\Sigma_{\text {sfr }}=10^{-1} \mathrm{M}_{\odot} \mathrm{yr}^{-1}$, similar to that predicted by star formation simulations in these systems (Sawala et al.2010).

For the star clusters embedded in the Antennae galaxy merging system, whose conditions are relatively well described by our model $\mathrm{A}$, we find $\Sigma_{\mathrm{sfr}} \approx 4 \times 10^{-2} \mathrm{M}_{\odot} \mathrm{yr}^{-1}$ per system, or a total rate of $\Sigma_{\mathrm{sfr}} \approx 4\left(N_{\mathrm{c}} / 10^{2}\right) \mathrm{M}_{\odot} \mathrm{yr}^{-1}$ for all $N_{\mathrm{c}}$ clusters. Such star formation rates were calculated assuming a star forming region of $5 \mathrm{pc}$ and a background ambient density of $\rho_{\infty}=10^{-21} \mathrm{~g} \mathrm{~cm}^{-3}$, which results in an average increase in cluster gas core density of $\langle\rho\rangle=10^{-18} \mathrm{~g} \mathrm{~cm}^{-3}$ (model A). The resulting rates are similar to those observed in the Antennae complex (Gilbert \& Graham 2007). If we assume that such rates are effectively sustained over the calculated orbital passage time scale of about 20 Myrs (Karl et al. 2010), one expects at least $10^{5} \mathrm{M}_{\odot}$ of new stars to be created per system. More detailed simulations that take into account self-gravity, realistic cooling and feedback effects are needed before detailed comparisons can be made with the inferred conditions required to explain subsequent episodes of star formation in stellar clusters (Conroy \& Spergel 2010, Gratton et al. 2004, Carretta et al. 2010; Piotto 2009;, Mackey et al. 2008).

\subsection{Accretion Onto Individual Stellar Members and the Central Massive Blackhole}

A compact star (or a black hole) of mass $M_{*}$, moving with relative velocity $u$ through a gas of ambient density $\rho_{\infty}$ and sound speed $c_{\infty}$, nominally accretes at the Bondi-HoyleLyttleton rate: $\dot{M} \approx 4 \pi\left(G M_{*}\right)^{2} \rho_{\infty}\left(u^{2}+c_{\infty}^{2}\right)^{-3 / 2}$. If, however, the collective potential of the system (e.g. star cluster or galaxy) was able to significantly increase the surrounding gas density prior to being accreted onto the individual neutron star members, then the accretion rates of the individual core stellar members (or the central black hole) would be enhanced by $\sim\langle\rho\rangle / \rho_{\infty}$, where $\langle\rho\rangle$ is the average increase in gas density in the core. It is to this problem that we now turn our attention. In particular, we study the effects of enhanced accretion rates onto the white dwarf core members and the central massive black hole.

\subsubsection{White Dwarfs in Stars Clusters and Galaxies}

In mature stellar systems, we expect a significant fraction of the stellar mass to be in white dwarfs. If the collective potential of the system (which contains the white dwarfs) is able to alter the ambient gas properties before the gas is accreted onto the individual stars, then the resulting enhanced

\footnotetext{
3 This is because the change in brightness across these two components is at least two orders of magnitude.
} 
accretion rates could significantly alter the state of the accreting stellar population. If, however, the density is not significantly increased, then the stars accrete gas as though they move through the external medium independently.

The resulting mass accretion rates for the white dwarf members depends on the radial distribution of both stars and gas. Here we calculate the expected mass accretion rates in the core of slowly moving star cluster (model A) using two extreme examples for the radial distribution of white dwarfs remnants. The first one is based on Fokker-Planck models of a core collapsed (centrally condensed) globular cluster (Dull et al. 1997), and the second simply assumes that the white dwarfs, containing $1 \%$ of the total mass, follow the radial stellar mass distribution. Figure 15 shows the mass accretion rate distribution of the white dwarf cluster members for both radial distributions (solid and dashed lines respectively).

This range of mass accretion rates are expected to result in enhanced novae rates (Nomoto et al. 2007). With a nova outburst rate of $\Psi_{\mathrm{nr}} \approx 10^{-4} \mathrm{yr}^{-1}$, we expect about $\approx 0.1\left(\Psi_{\mathrm{nr}} / 10^{-4} \mathrm{yr}^{-1}\right)\left(N_{\mathrm{wd}} / 10^{4}\right)\left(N_{\mathrm{c}} / 10^{2}\right)$ outbursts per year. This is probably a conservative estimate as the individual novae rates are expected to increase to $\Psi \leq 10^{-1} \mathrm{yr}^{-1}$ for the larger accretion rates calculated here (Starrfield et al. 1985). These novae outbursts will be observed as super soft X-ray sources $(k T \leq 1 \mathrm{keV})$ with luminosities $\lesssim 10^{38} \mathrm{erg} \mathrm{s}^{-1}$ and thus might be observable in nearby merging galaxies (Hernanz \& Sala 2010).

Such high mass accretion rates can only be sustained if star clusters move slowly with respect to the background gas. While there are no stellar clusters observed in the Galactic disk which bear these anticipated properties (the relative velocity of the halo clusters to the interstellar medium is in the range of $100 \mathrm{~km} \mathrm{~s}^{-1}$ ), observations of several cluster knots in the Antennae indicate intracluster relative velocities that comparable to the central velocity dispersions. In these systems, it has been shown that this merging state is, however, short lived, $\Delta t \lesssim 20 \mathrm{Myr}$ (Karl et al. 2010). Even if this (slowly moving) cold gas phase persists for $\leq 20 \mathrm{Myr}$, we expect at least $30 \%$ of the white dwarf members to double their mass. This increase in mass would in turn lead to a sizable increase in luminosity (e.g. Mestel 1952), which in turn modify the white dwarf cooling sequence.

\subsubsection{Accretion onto Central Massive Black Holes}

Most early-type galaxies and spiral bulges are now thought to contain massive black holes in their nuclei (Magorrian et al. 1998). Direct evidence is also now available which supports the idea that active galaxies are powered by massive black holes. It can then be argued that many if not all galaxies could be expected to have undergone an active phase and to possess a central massive black hole (Haehnelt \& Rees 1993), although the presence of intermediate mass black holes in dwarf galaxies (and some globular clusters) remains controversial (Ulvestad et al. 2007, Gebhardt et al. 2005; Baumgardt et al. 2003a).

Large density enhancements in the nuclei of galaxies, dwarf galaxies and star clusters would be accompanied by enhanced mass supply to the central black hole. For a typical galactic halo (model E), this enhanced mass accretion results in a significant black hole accretion luminosity:

$$
L_{\mathrm{bh}} \approx 10^{41} \frac{M_{\mathrm{bh}}}{10^{6} M_{\odot}} \frac{\epsilon}{0.1} \operatorname{erg~s}^{-1},
$$

where $\epsilon$ is the efficiency for converting gravitational energy into radiation. Extrapolating the $M_{\mathrm{bh}}-\sigma$ relation (Tremaine et al. 2002) to the star clusters and dwarf galaxies modeled by simulations [A, B, C, D] gives black hole masses of $M_{\mathrm{bh}}=[0.68,0.68,1.42,1.42] \times 10^{4} M_{\odot}$ and accretion luminosities of $\log L_{x} / L_{\text {edd }}=\left[10^{1.77}, 10^{-1.46}, 10^{2.18}, 10^{-6.20}\right]$, respectively. While the integrated output from this increased mass feeding could, in principle, amount to large factors of the black hole's Eddington luminosity, it would probably be significantly less because we have, for simplicity, neglected feedback.

We thank Thorsten Naab, Elena D'Onghia, Charlie Conroy, Aaron Romanowsky, Jürg Diemand, and Lars Hernquist for useful discussions. We also thank the referee for his/her insightful comments. The software used in this work was in part developed by the DOE-supported ASCI/Alliance Center for Astrophysical Thermonuclear Flashes at the University of Chicago. Computations were performed on the Pleaides UCSC computer cluster. This work is supported by NSF: AST-0847563 (J.N. and E.R.), NASA: NNX08AL41G (J.N. and D.L.), and The David and Lucile Packard Foundation (E.R.). 


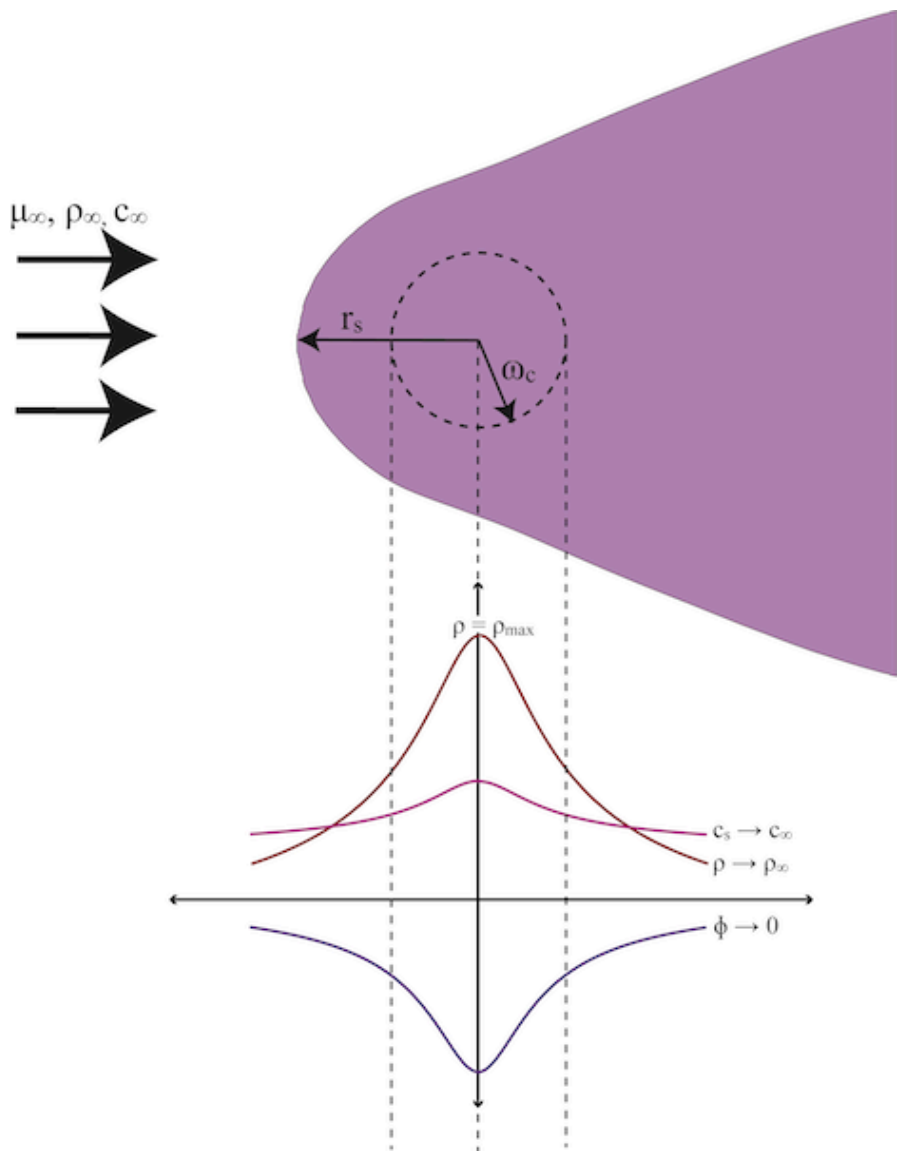

FIG. 1.- Diagram shows the flow geometry of non-stationary core potentials with central mass accumulation. A bow shock is produced by the motion of the potential with respect to the ambient gas. The initial asymptotic Mach number, sound speed and density of the inflowing gas are denoted here as $\mu_{\infty}, c_{\infty}$ and $\rho_{\infty}$, respectively. Also depicted are the characteristic, $r_{\mathrm{s}}$, and the core, $\Phi_{\mathrm{c}}$, radii, together with a sketch of the expected profiles of density, $\rho(r)$, and sound speed, $c_{\mathrm{s}}(r)$, along the flow axis.

\section{REFERENCES}

Adén, D., Wilkinson, M. I., Read, J. I., Feltzing, S., Koch, A., Gilmore,

G. F., Grebel, E. K., \& Lundström, I. 2009, ApJ, 706, L150

Allen, S. W., Ettori, S., \& Fabian, A. C. 2001, MNRAS, 324, 877

Baumgardt, H., Hut, P., Makino, J., McMillan, S., \& Portegies Zwart, S.

2003, ApJ, 582, L21

Belokurov, V., et al. 2007, ApJ, 654, 897

Blondin, J. M., \& Pope, T. C. 2009, ApJ, 700, 95

Bondi, H. 1952, MNRAS, 112, 195

Brandl, B. R., et al. 2005, ApJ, 635, 280

Brüns, R. C., Kroupa, P., \& Fellhauer, M. 2009, ApJ, 702, 1268

Carretta, E., Bragaglia, A., Gratton, R. G., Recio-Blanco, A., Lucatello, S. D’Orazi, V., \& Cassisi, S. 2010, A\&A, 516, A55

Chung, S. M., Gonzalez, A. H., Clowe, D., Zaritsky, D., Markevitch, M., \& Jones, C. 2009, ApJ, 691, 963

Conroy, C., \& Spergel, D. N. 2010, arXiv:1005.4934

Crivellari, E., Wolter, A., \& Trinchieri, G. 2009, A\&A, 501, 445

Diemand, J., Kuhlen, M., \& Madau, P. 2007, ApJ, 667, 859

Diemand, J., Kuhlen, M., Madau, P., Zemp, M., Moore, B., Potter, D., \& Stadel, J. 2008, Nature, 454, 735

Dinescu, D. I., Girard, T. M., van Altena, W. F., Mendez, R. A., \& Lopez, C. E. 1997, AJ, 114, 1014

Dull, J. D., Cohn, H. N., Lugger, P. M., Murphy, B. W., Seitzer, P. O., Callanan, P. J., Rutten, R. G. M., \& Charles, P. A. 1997, ApJ, 481, 267

Edgar, R. 2004, New Astronomy Review, 48, 843

Fryxell, B., et al. 2000, ApJS, 131, 273

Gebhardt, K., Rich, R. M., \& Ho, L. C. 2005, ApJ, 634, 1093

Gilbert, A. M., \& Graham, J. R. 2007, ApJ, 668, 168
Gratton, R., Sneden, C., \& Carretta, E. 2004, ARA\&A, 42, 385

Harris, W. E. 1991, ARA\&A, 29, 543

Harris, W. E. 1996, AJ, 112, 1487

Haehnelt, M. G., \& Rees, M. J. 1993, MNRAS, 263, 168

Hayashi, E., Navarro, J. F., \& Springel, V. 2007, MNRAS, 377, 50

Hernanz, M., \& Sala, G. 2010, Astronomische Nachrichten, 331, 169

Hernquist, L. 1990, ApJ, 356, 359

Hwang, H. S., \& Lee, M. G. 2008, ApJ, 676, 218

Karl, S. J., Naab, T., Johansson, P. H., Kotarba, H., Boily, C. M., Renaud, F., \& Theis, C. 2010, ApJ, 715, L88

Kennicutt, R. C., Jr. 1998, ApJ, 498, 541

Knapp, G. R., Gunn, J. E., Bowers, P. F., \& Vasquez Poritz, J. F. 1996, ApJ, 462,231

Lin, D. N. C., \& Murray, S. D. 2007, ApJ, 661, 779

Mackey, A. D., Broby Nielsen, P., Ferguson, A. M. N., \& Richardson, J. C. 2008, ApJ, 681, L17

Magorrian, J., et al. 1998, AJ, 115, 2285

Markevitch, M., Gonzalez, A. H., David, L., Vikhlinin, A., Murray, S.,

Forman, W., Jones, C., \& Tucker, W. 2002, ApJ, 567, L27

Markevitch, M. 2006, The X-ray Universe 2005, 604, 723

Mateo, M. L. 1998, ARA\&A, 36, 435

Meiksin, A. A. 2009, Reviews of Modern Physics, 81, 1405

Mestel, L. 1952, MNRAS, 112, 583

Milosavljević, M., Koda, J., Nagai, D., Nakar, E., \& Shapiro, P. R. 2007, ApJ, 661, L131

Naiman, J. P., Ramirez-Ruiz, E., \& Lin, D. N. C. 2009, ApJ, 705, L153

Nomoto, K., Saio, H., Kato, M., \& Hachisu, I. 2007, ApJ, 663, 1269 
TABLE 1

AstrophysiCally Motivated Simulations

\begin{tabular}{|c|c|c|c|c|c|c|c|c|c|c|}
\hline $\begin{array}{c}\text { Simulation } \\
\text { Label }\end{array}$ & $\gamma$ & $\begin{array}{c}c_{\infty} \\
{\left[\mathrm{km} \mathrm{s}^{-1}\right]}\end{array}$ & $\mu_{\infty}$ & $\begin{array}{c}\bar{\varpi}_{\mathrm{c}} \\
{[\mathrm{pc}]}\end{array}$ & $\begin{array}{c}M_{p} \text { or } M_{h} \\
{\left[\mathrm{M}_{\odot}\right]}\end{array}$ & $\begin{array}{c}\text { Analytic } \\
\log \left(\rho_{\max } / \rho_{\infty}\right)\end{array}$ & $\begin{array}{c}\text { Simulated } \\
\log \left(\rho_{\max } / \rho_{\infty}\right)\end{array}$ & $\begin{array}{c}\text { Analytic } \\
r_{\mathrm{s}}[\mathrm{pc}]\end{array}$ & $\begin{array}{c}\text { Simulated } \\
r_{\mathrm{s}}[\mathrm{pc}]\end{array}$ & $u_{\max } / c_{\infty}$ \\
\hline $\mathrm{A}^{\mathrm{a}}$ & 1.01 & 10 & 2 & 1 & $3.5 \times 10^{5}$ & 6.03 & 4.32 & 2.7 & 1.7 & 3.8 \\
\hline $\mathrm{B}^{\mathrm{b}}$ & 1.01 & 10 & 4 & 2 & $3.5 \times 10^{5}$ & 3.12 & 1.08 & 1.34 & $0^{\dagger}$ & 5.3 \\
\hline $\mathrm{C}^{\mathrm{c}}$ & 1.01 & 10 & 2 & 200 & $10^{8}$ & 8.38 & 4.79 & 1500 & 210 & 4.8 \\
\hline $\mathrm{D}^{\mathrm{d}}$ & 1.67 & 100 & 4 & 200 & $10^{8}$ & 0.09 & 0.03 & 11 & $0^{\dagger}$ & 4 \\
\hline $\mathrm{E}^{\mathrm{e}}$ & 1.67 & 100 & 2 & $3 \times 10^{4}$ & $8 \times 10^{11}$ & 1.5 & 0.77 & $1.5 \times 10^{5}$ & $1.4 \times 10^{4}$ & 3.23 \\
\hline$F^{f}$ & 1.67 & 1580 & 2.5 & $10^{6}$ & $10^{15}$ & 0.5 & 0.86 & $6 \times 10^{5}$ & $3.6 \times 10^{5}$ & 4.4 \\
\hline
\end{tabular}

a Young star cluster modeled here by a Plummer potential.

b Globular cluster modeled by a Plummer potential.

c Slowly moving dwarf galaxy in cool gas modeled by a Hernquist potential.

d Slowly moving dwarf galaxy in hot gas modeled by a Hernquist potential.

e Milky Way sized halo moving through hot gas modeled by a Hernquist potential.

f Simulation of galaxy cluster similar to the Bullet Cluster, modeled by a Hernquist potential.

$\dagger$ These simulations resulted in a detached tailshock.

Peres, G., Serio, S., Vaiana, G. S., \& Rosner, R. 1982, ApJ, 252, 791

Pflamm-Altenburg, J., \& Kroupa, P. 2009, MNRAS, 397, 488

Piotto, G. 2009, IAU Symposium, 258, 233

Plummer, H. C. 1911, MNRAS, 71, 460

Robaina, A. R., Bell, E. F., van der Wel, A., Somerville, R. S., Skelton, R. E., McIntosh, D. H., Meisenheimer, K., \& Wolf, C. 2010, ApJ, 719, 844

Rosner, R., Tucker, W. H., \& Vaiana, G. S. 1978, ApJ, 220, 643

Ruffert, M. 1994, A\&AS, 106, 505

Sawala, T., Guo, Q., Scannapieco, C., Jenkins, A., \& White, S. D. M. 2010, arXiv:1003.0671

Scott, N., et al. 2009, MNRAS, 398, 1835

Shu, F. H. 1992, The physics of astrophysics. Volume II: Gas dynamics., by Shu, F. H.. University Science Books, Mill Valley, CA (USA), 1992, 493 p., ISBN 0-935702-65-2, Price
Starrfield, S., Sparks, W. M., \& Truran, J. W. 1985, ApJ, 291, 136 Tremaine, S., et al. 2002, ApJ, 574, 740

Ulvestad, J. S., Greene, J. E., \& Ho, L. C. 2007, ApJ, 661, L151

Whitmore, B. C., \& Schweizer, F. 1995, AJ, 109, 960

Whitmore, B. C., Zhang, Q., Leitherer, C., Fall, S. M., Schweizer, F., \&

Miller, B. W. 1999, AJ, 118, 1551

Whitmore, B. C., et al. 2005, AJ, 130, 2104

Wilkin, F. P. 1996, ApJ, 459, L31

Wolfson, R. 1977, ApJ, 213, 200

Zhu, M., Seaquist, E. R., \& Kuno, N. 2003, ApJ, 588, 243 

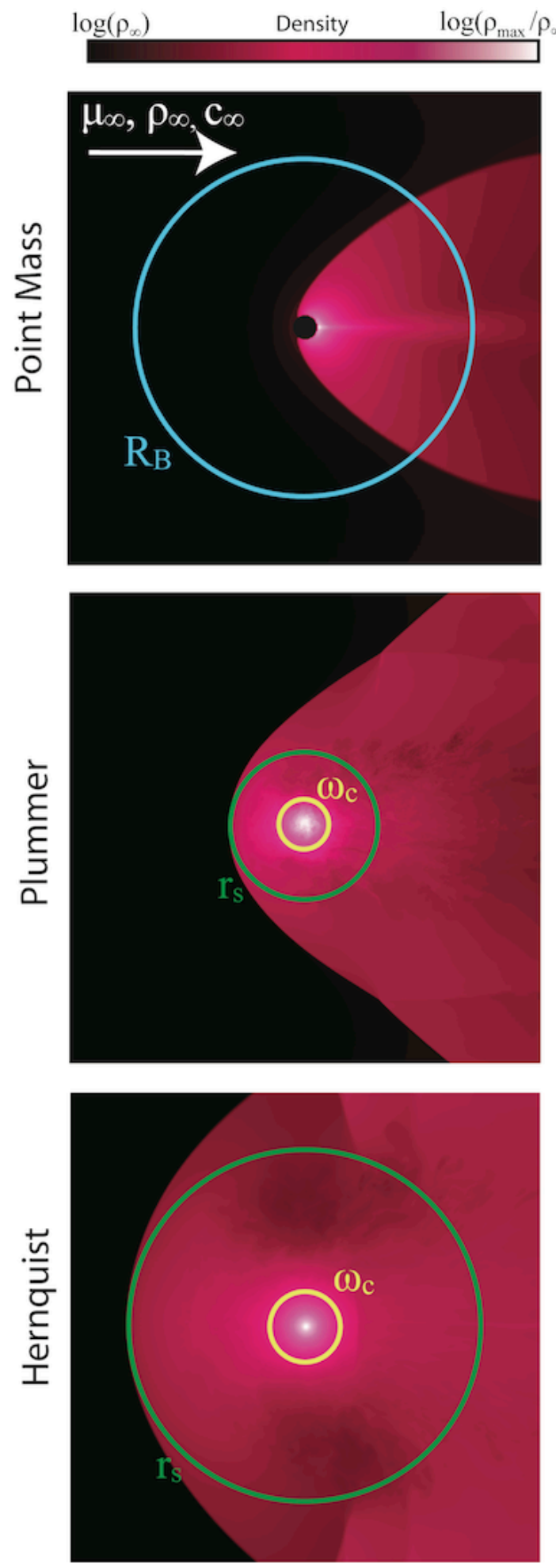

FIG. 2.- Density contours of the flow pattern around point mass, Plummer and Hernquist potentials set in motion through an initially uniform medium with $\rho_{\infty}=1$ and $\mu_{\infty}=2$. The point mass potential is implemented here with a sink boundary following the method of Ruffert (1994) by flooring the values of density and pressure to $50 \%$ of their background values and setting the velocities to zero inside the sink radius. Color bars show density cuts through the $x y$-plane with limiting values: $\log \left(\rho_{\max } / \rho_{\infty}\right)=[1.2,1.1,1.5]$ for point mass, Plummer and Hernquist potentials, respectively. The equation of state used here is adiabatic $(\gamma=5 / 3)$. Also depicted are the characteristic, $r_{\mathrm{S}}$ (which reduces to the Bondi radius $R_{\mathrm{B}}$ for a point mass potential), and the core, $\Phi_{\mathrm{c}}$, radii. The Plummer and Hernquist potentials have been constructed to yield the same circular velocity peak radius: $r_{\mathrm{v}}$. Note that the difference between $r_{\mathrm{s}}$ in the Plummer and Hernquist potentials is consistent with equation 30 . 


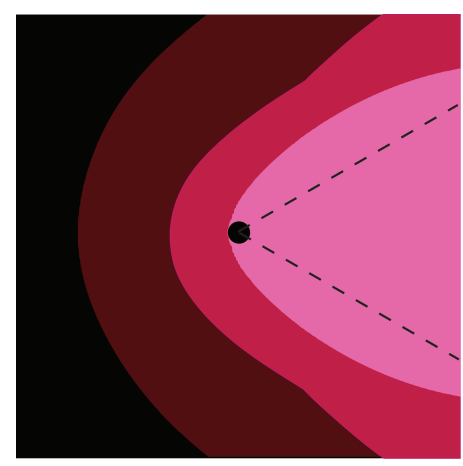

FIG. 3.- Bow shock patterns produced around a point mass (pink), Plummer (red) and Hernquist (brown) potentials set in motion through an initially uniform medium with $\mu_{\infty}=2.0$. Also plotted, for comparison, is the bow shock expected for a supersonically moving, non-gravitating object (dashed line). In this case the bow shock's opening angle is simply given by $\theta_{\text {bow }}=\arcsin \mu_{\infty}^{-1}$. The size of the box is 40 times the sink size (used here to model the point mass potential), depicted by the black circle. 


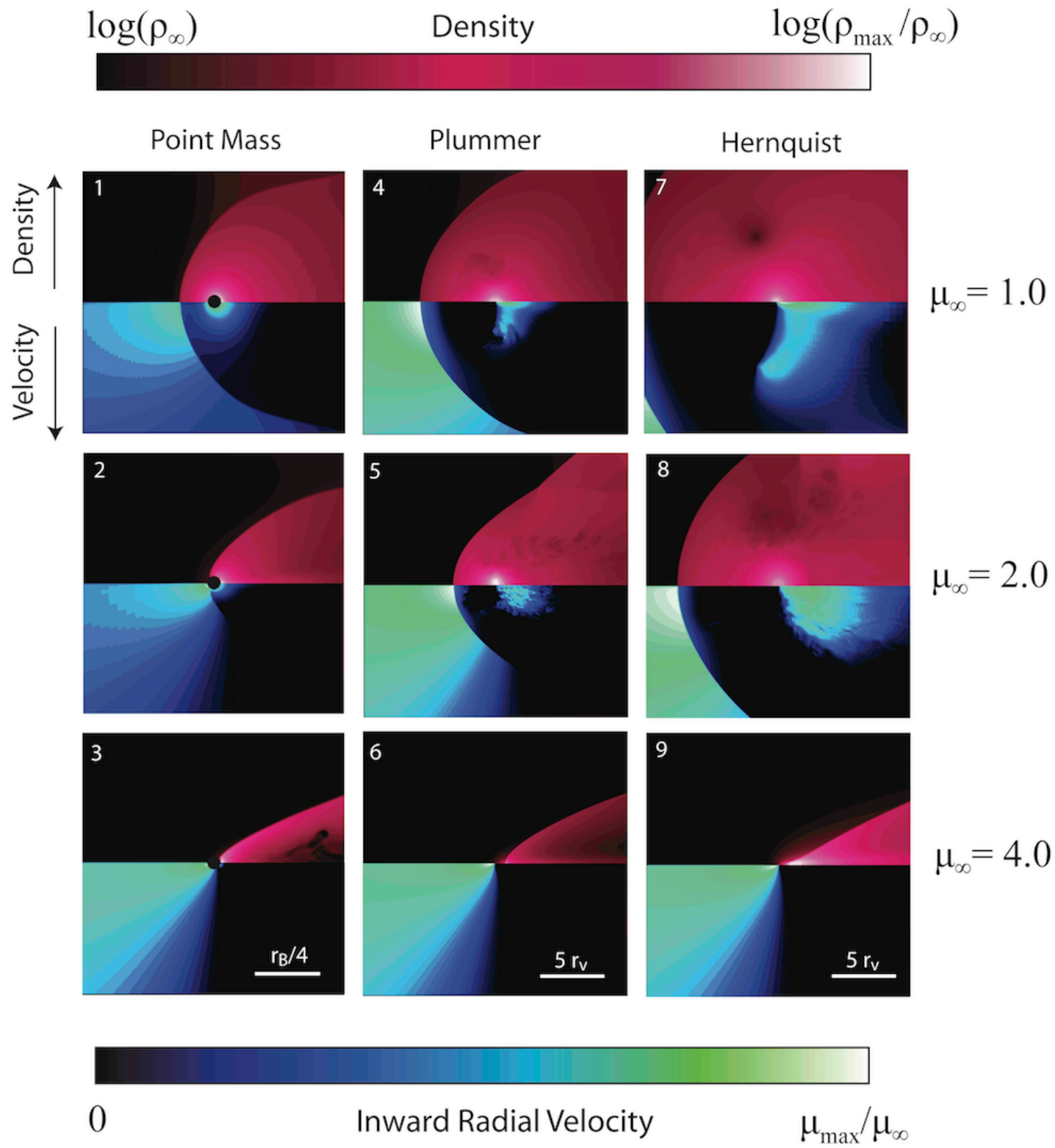

FIG. 4.- Density and radial velocity contours for gas flowing around a point mass, Plummer and Hernquist potential at various relative velocities. We exploit the flow symmetries to plot both the density contrast (upper, maroon half) and inward radial velocity profiles (lower, blue half) in the same panel. Color bars show density and velocity cuts through the $x y$-plane in units of $\log \left(\rho_{\max } / \rho_{\infty}\right)$ and $\mu / \mu_{\infty}$. The maximum densities and radial velocities attained in simulations 1 through 9 are $\log \left(\rho_{\max } / \rho_{\infty}\right)=[1.5,1.2,0.8,1.4,1.1,1.4,1.7,1.5,1.1]$ and $\left(\mu_{\max } / \mu_{\infty}\right)=[3.9,6.2,6.9,1.9,3.1,6.2,1.7,2.9,6.4]$, respectively. The equation of state used here is adiabatic $(\gamma=5 / 3)$. 


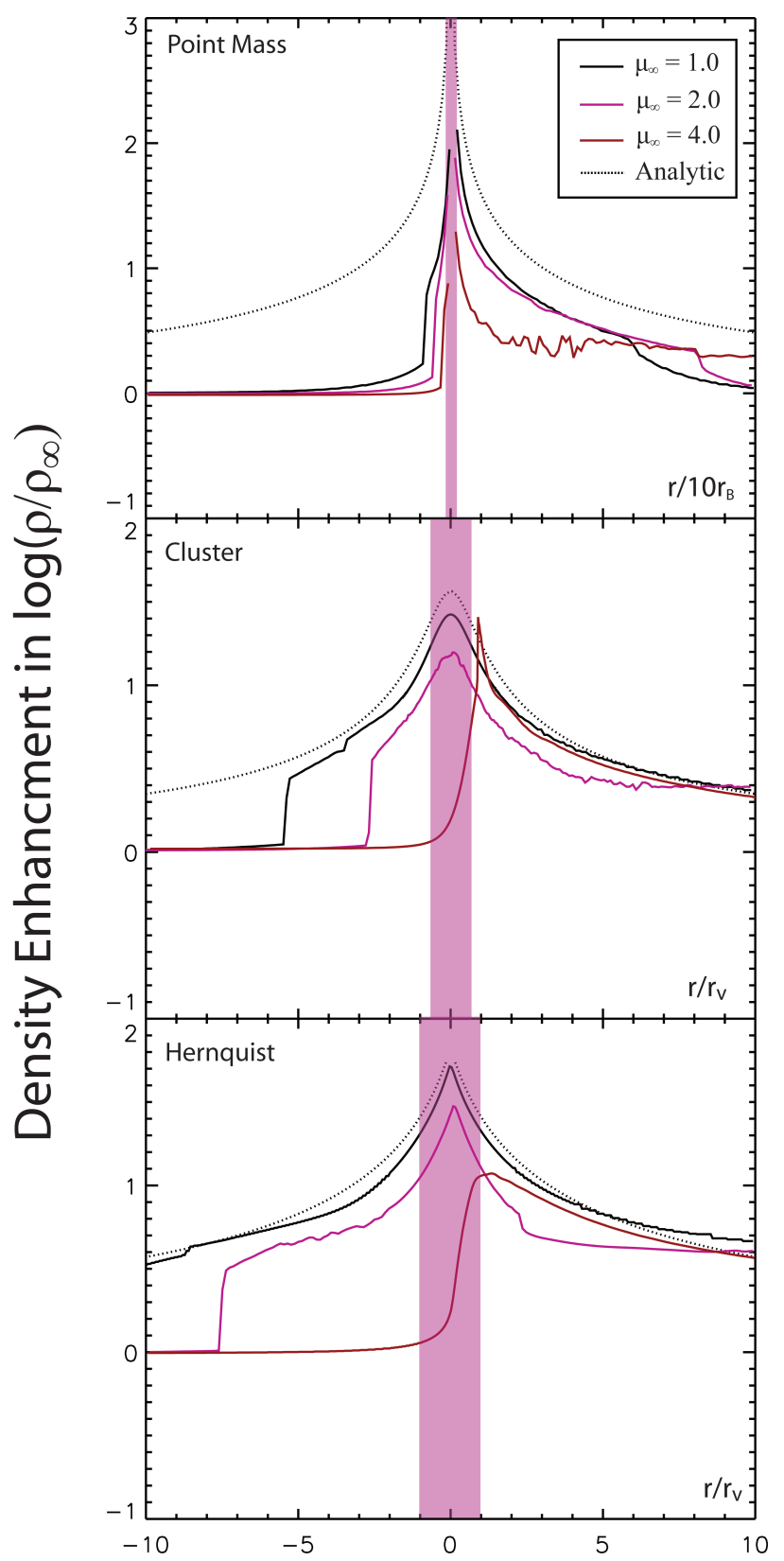

FIG. 5.- Density cuts along the axis parallel to the incoming flow for the simulations shown in Figure 4 The dotted lines give the analytic solutions for a point mass, Plummer and Hernquist potential, while the black, pink and red lines show the numerical profiles for $\mu_{\infty}=1.0,2.0$, and 4.0, respectively. The shaded regions depict either the core radii or the sink size. 


\section{Density}

\section{$\log \left(\rho_{\infty}\right)$ \\ $\log \left(\rho_{\max } / \rho_{\infty}\right)$}

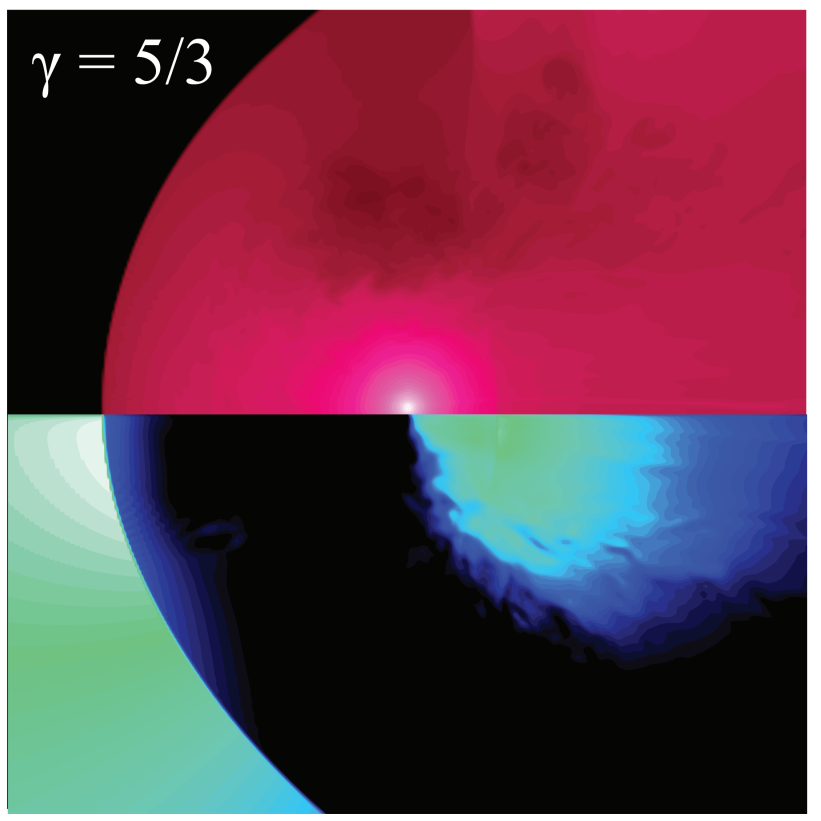

$$
\gamma=1.01
$$

$$
\begin{gathered}
\mu_{\infty}=2.0 \\
5 r_{v}
\end{gathered}
$$

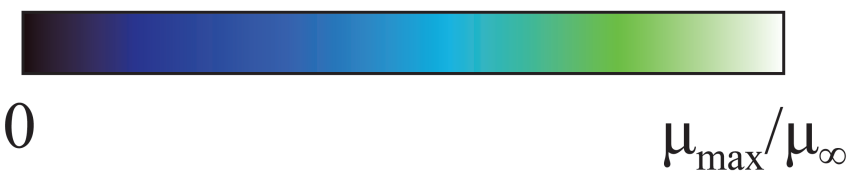

\section{Inward Radial Velocity}

FIG. 6.-Density and radial velocity contours for gas flowing with $\mu_{\infty}=$ 2.0 around a Hernquist potential in the adiabatic $(\gamma=5 / 3)$ and isothermal $(\gamma=1.01)$ limits. The maximum densities and radial velocities attained in simulations for $\gamma=5 / 3$ and $\gamma=1.01$ are $\log \left(\rho_{\max } / \rho_{\infty}\right)=[1.5,5.1]$ and $\left(\mu_{\max } / \mu_{\infty}\right)=[2.9,4.9]$, respectively. 


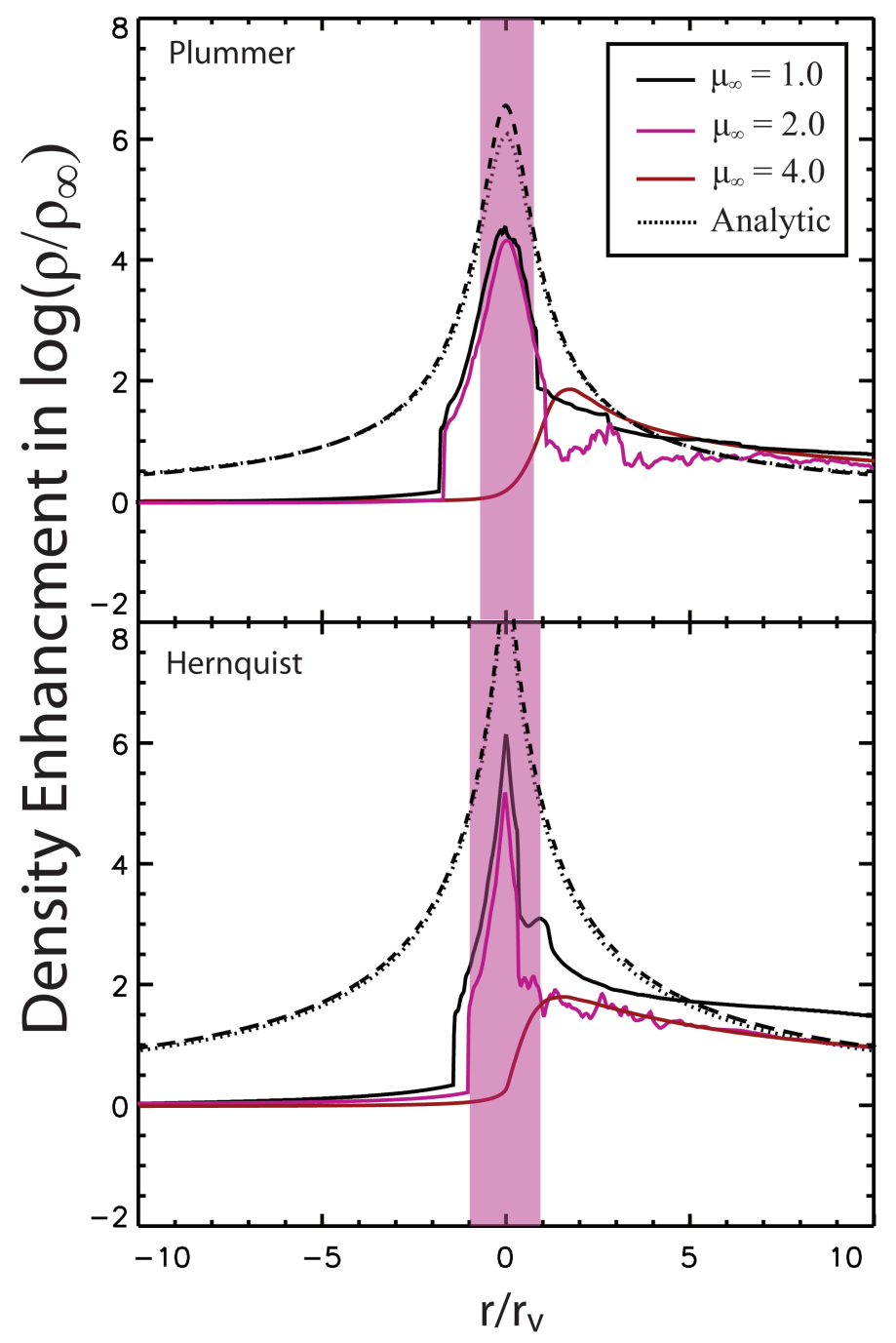

FIG. 7.- Same as Figure 5 but for a near isothermal equation of state $(\gamma=1.01)$. The dotted lines are the analytic profiles derived in $\$ 2$ specifically equations 23 and 25$]$ with $\gamma=1.01$. The dashed lines are the isothermal form of the density equation, (11). The shaded regions show the size of the core. 

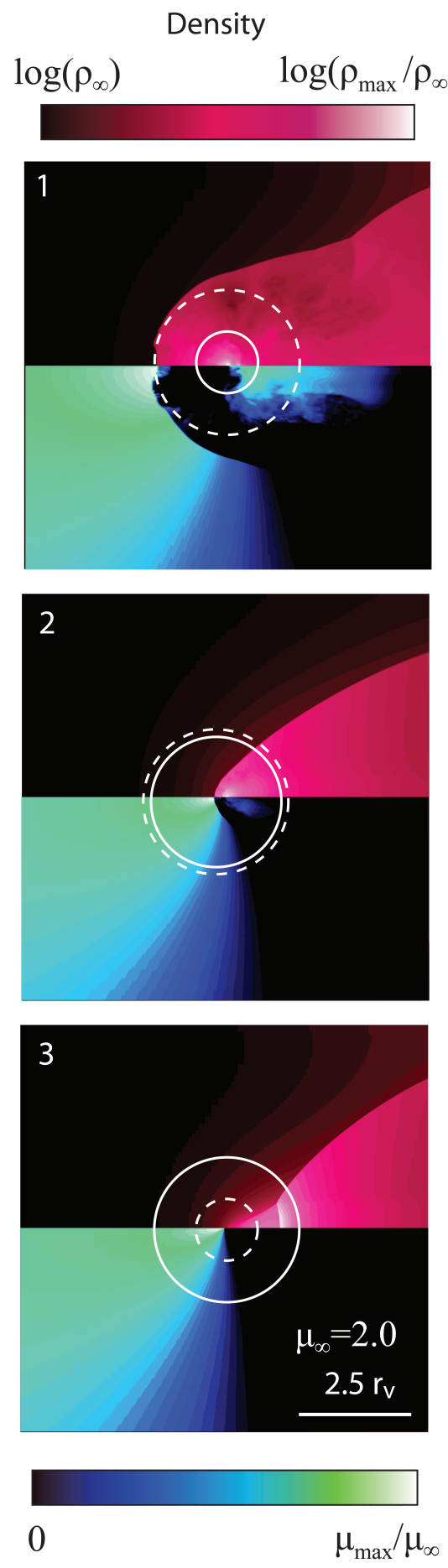

\section{Inward Radial Velocity}

FIG. 8.- Density and radial velocity contours for gas flowing with $\mu_{\infty}=$ 2.0 around a Hernquist potential with varying $r_{\mathrm{s}}$ in the adiabatic $(\gamma=5 / 3)$ limit. Dashed circles depict the location of the analytic characteristic radius, $r_{\mathrm{s}}$ (equations [19] and [20]), while solid circles give the approximate location of the core radius $\varpi_{\mathrm{c}}$. The maximum densities and radial velocities attained in simulations 1 through 6 are $\log \left(\rho_{\max } / \rho_{\infty}\right)=[1.8,1.3,1.0]$ and $\left(\mu_{\max } / \mu_{\infty}\right)=$ $[3.3,3.6,3.2]$, respectively. 
Naiman et al.

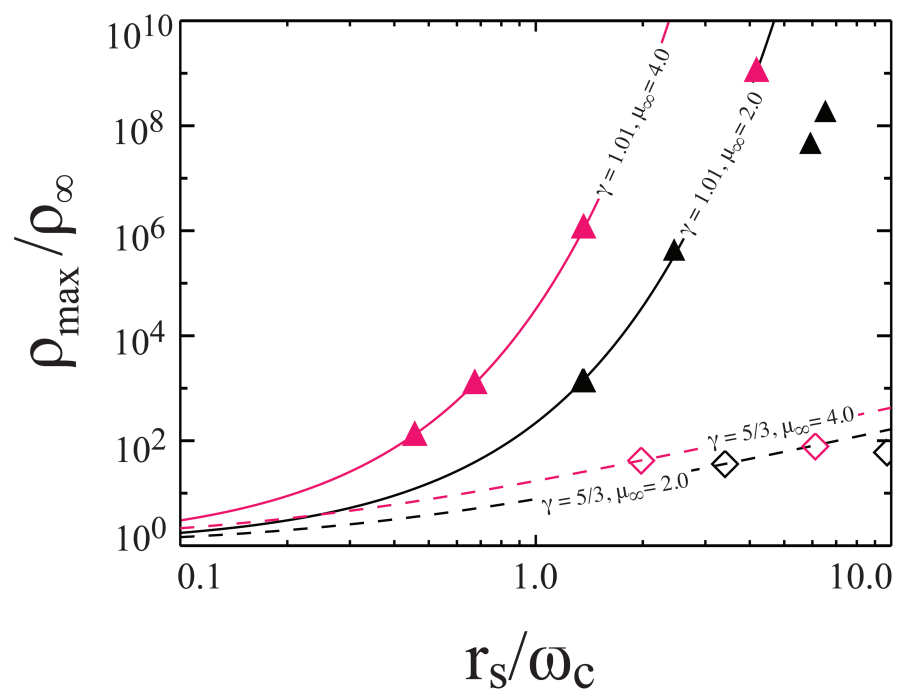

FIG. 9.- Expected density enhancements as a function of $r_{\mathrm{s}} / \varpi_{\mathrm{c}}$ for a variety of simulations with $c_{\infty}=10 \mathrm{~km} \mathrm{~s}^{-1}$. The triangles (diamonds) are measured $\rho_{\max } / \rho_{\infty}$ and $r_{\mathrm{s}} / \varpi_{\mathrm{c}}$ from the isothermal (adiabatic) simulations. For comparison, the lines are trends of $\rho_{\max } / \rho_{\infty}$ from the analytic results from equations 20) and 23 for Plummer potentials with a variety of masses and core radii in $c_{\infty}=10 \mathrm{~km} \mathrm{~s}^{-1}$ gas. 


\section{$\log \left(\rho_{\infty}\right) \quad$ Density $\log \left(\rho_{\max } / \rho_{\infty}\right)$}
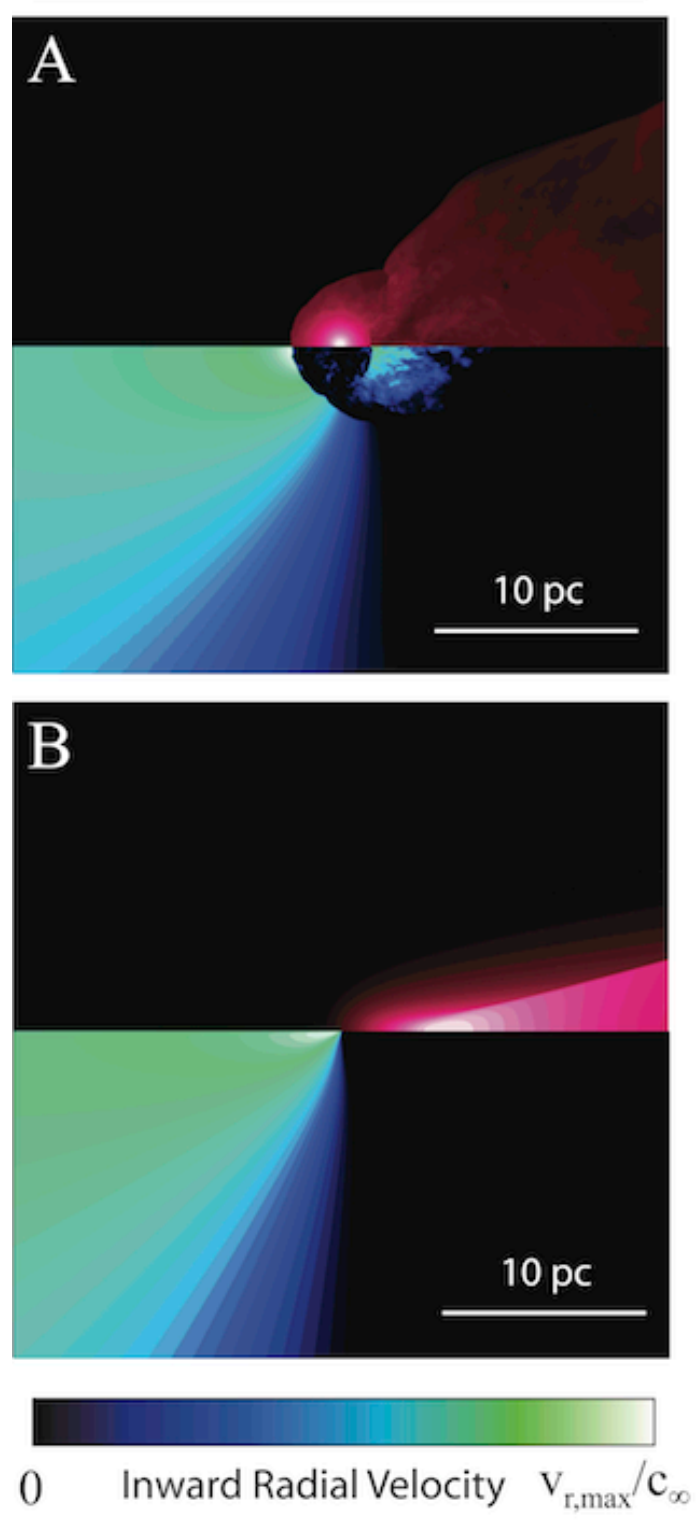

FIG. 10.- Examples of gas accumulation in star clusters. Density and radial velocity contours for models $\mathrm{A}$ and $\mathrm{B}$. The maximum densities and radial velocities attained in the simulations are $\log \left(\rho_{\max } / \rho_{\infty}\right)=[4.32,1.08]$ and $\left(u_{\max } / c_{\infty}\right)=[3.8,5.3]$, respectively. 


\section{$\log \left(\rho_{\infty}\right) \quad$ Density $\log \left(\rho_{\max } / \rho_{\infty}\right)$}


FIG. 11.- Examples of gas accumulation in dwarf galaxies. Density and radial velocity contours for models $\mathrm{C}$ and $\mathrm{D}$. The maximum densities and radial velocities attained in the simulations $\operatorname{are} \log \left(\rho_{\max } / \rho_{\infty}\right)=[4.79,0.03]$ and $\left(u_{\max } / c_{\infty}\right)=[4.8,4.0]$, respectively. 


\section{$\log \left(\rho_{\infty}\right) \quad$ Density $\log \left(\rho_{\max } / \rho_{\infty}\right)$}


0 Inward Radial Velocity $\quad \mathrm{V}_{\mathrm{r}, \max } / \mathrm{c}_{\infty}$

FIG. 12.- Gas accumulation for a Milky Way-like halo moving in a major merging environment. Density and radial velocity contours for model $\mathrm{E}$. The maximum densities and radial velocities attained in the simulation are $\log \left(\rho_{\max } / \rho_{\infty}\right)=0.77$ and $\left(u_{\max } / c_{\infty}\right)=3.23$, respectively. 


\section{$\log \left(\rho_{\infty}\right) \quad$ Density $\log \left(\rho_{\max } / \rho_{\infty}\right)$}
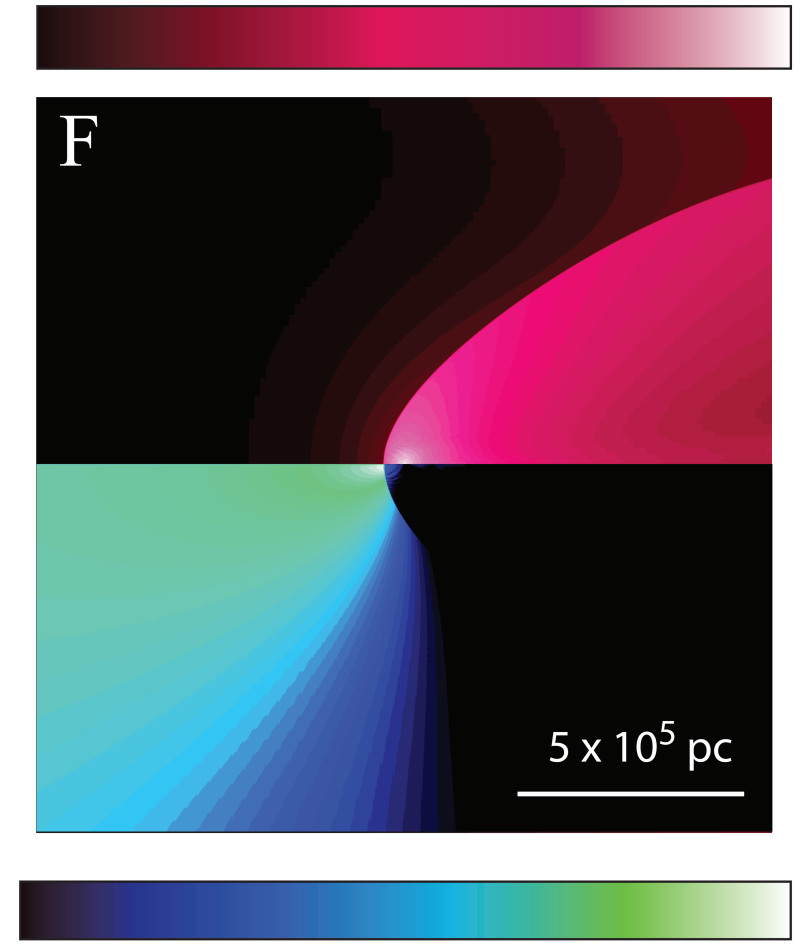

$0 \quad$ Inward Radial Velocity $\quad \mathrm{V}_{\mathrm{r}, \max } / \mathrm{c}_{\infty}$

FIG. 13.- Gas accumulation for a galaxy cluster in a major merger environment. Density and radial velocity contours for model F. The maximum densities and radial velocities attained in the simulation are $\log \left(\rho_{\max } / \rho_{\infty}\right)=[0.86]$ and $\left(u_{\max } / c_{\infty}\right)=[4.4]$, respectively. 

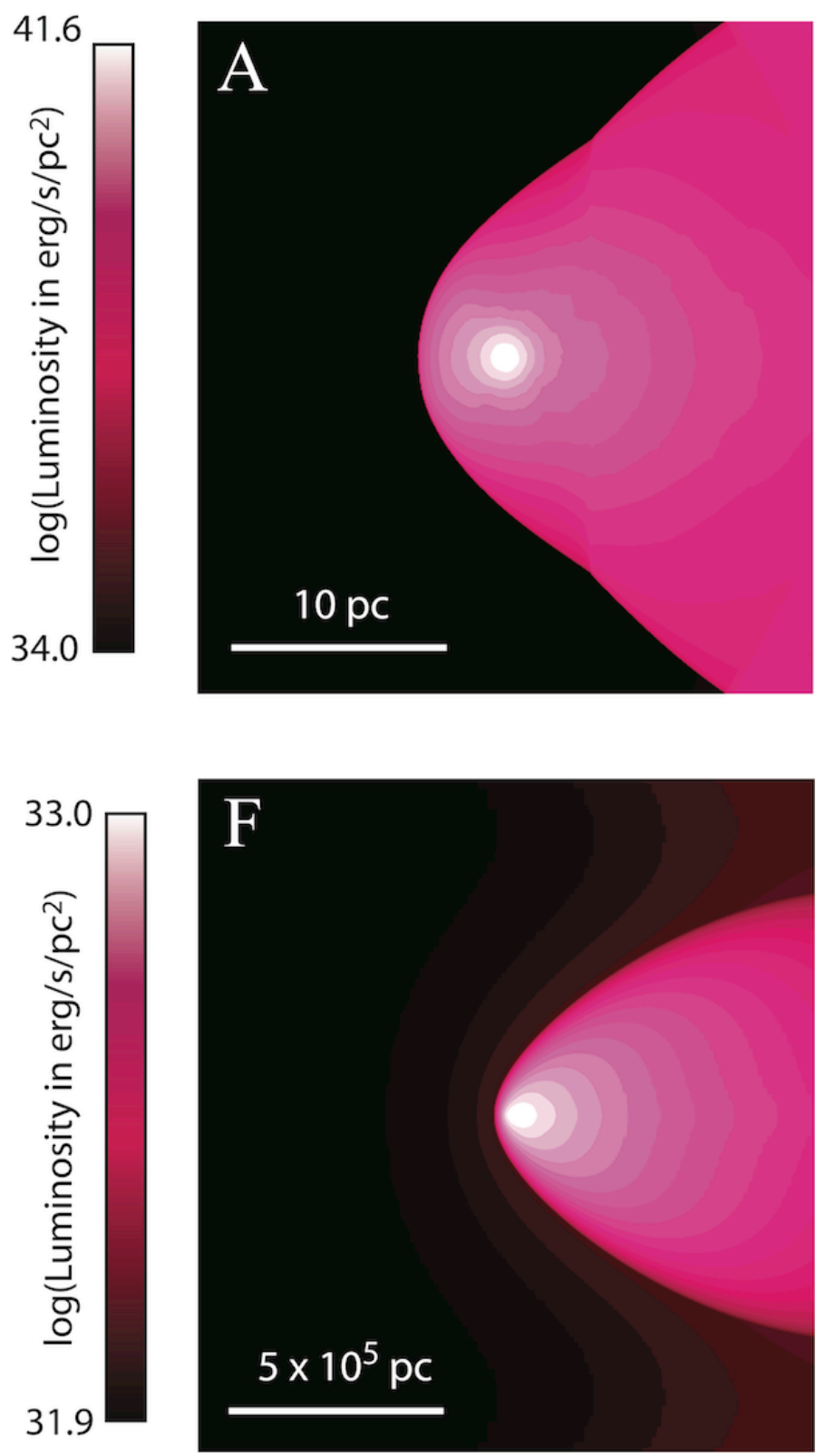

FIG. 14.- The emission properties of the accumulated gas in a galaxy cluster (model F) and a young stellar cluster (model A). Emission is calculated using the cooling curves for an optically thin plasma from Peres et al. (1982) for chromospheric energy losses in the temperature range $4.44 \times 10^{3}<T<$ $2 \times 10^{4} \mathrm{~K}$, the curves of Rosner et al. (1978) for coronal temperatures of $2 \times 10^{4}<T<10^{8} \mathrm{~K}$, and bremsstrahlung emission above $10^{8} \mathrm{~K}$. We assume $\gamma=5 / 3$. That is, the cooling term is not explicitly included in the energy equation. The ionization fraction is assumed to be purely collisional, giving $\chi_{i}=0.003$ for model A and $\chi_{i} \sim 1$ for model F. Model A has a temperature range in the band $k T=0.6-7.2 \mathrm{eV}$, while for model $\mathrm{F}$ the range is $k T=15.0-146.0 \mathrm{keV}$. 


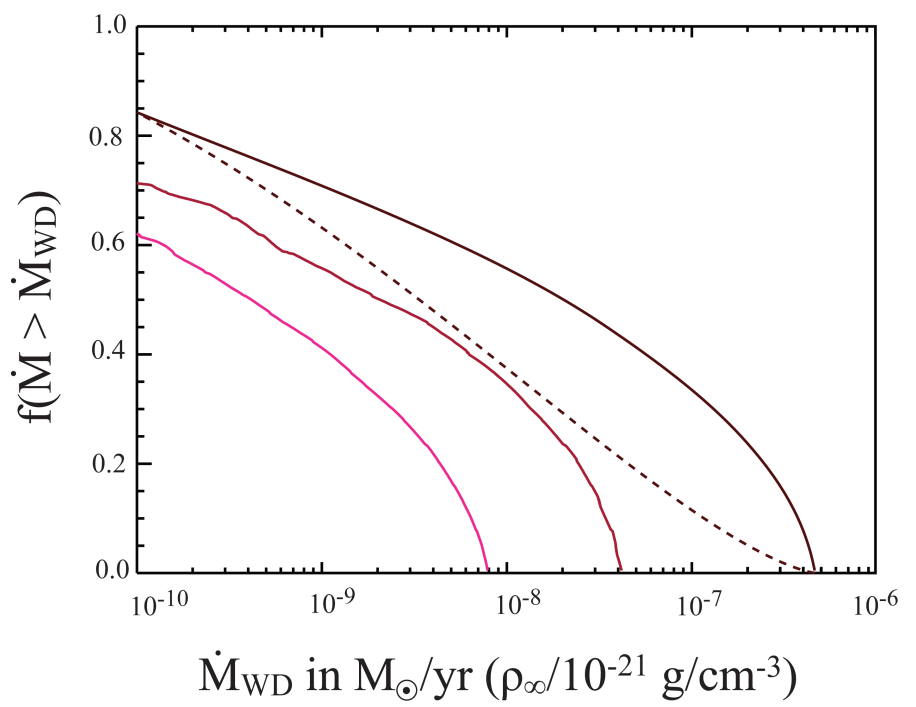

FIG. 15.- The mass accretion rate fraction, $f$, of the white dwarf cluster members for both centrally condensed (solid line) and non-condensed (dashed line) compact remnant distributions. The gas density profile for model A has been used, with $\rho_{\infty}=10^{-21} \mathrm{~g} \mathrm{~cm}^{-3}$. To calculate the mass accretion distributions we have assumed $M_{\mathrm{wd}}=1 M_{\odot}$ and $u_{\mathrm{wd}}=15 \mathrm{~km} \mathrm{~s}^{-1}$. The red (brown) line was calculating using the numerical density profile for $\mu_{\infty}=2.0(0.5)$. The black line assumes the analytic profile for $\mu_{\infty}=2.0$. 